\section{Design Principles of the ESCRT-III Vps24-Vps2 Module} Sudeep Banjade ${ }^{1,2}$, Yousuf H. Shah ${ }^{1,2}$, Shaogeng Tang ${ }^{3}$, Scott D. Emr ${ }^{1,2}$ *

${ }^{1}$ Weill Institute for Cell and Molecular Biology, Cornell University, Ithaca, NY, USA

${ }^{2}$ Department of Molecular Biology and Genetics, Cornell University, Ithaca, NY, USA

${ }^{3}$ Department of Biochemistry, Stanford University, Stanford, CA, USA

*Correspondence: sde26@cornell.edu

\title{
Abstract
}

ESCRT-III polymerization is required for all ESCRT-dependent events in the cell. However, the relative contributions of the eight ESCRT-III subunits differ between each process. The minimal features of ESCRT-III proteins necessary for function, and the role for the multiple ESCRT-III subunits remain unclear. To identify essential features of ESCRT-III subunits, we previously studied the polymerization mechanisms of two ESCRT-III subunits Snf7 and Vps24, identifying the association of the helix-4 region of Snf7 with the helix-1 region of Vps24 (Banjade et al., 2019). Here, we find that mutations in the helix-1 region of another ESCRT-III subunit Vps 2 can functionally replace Vps24 in S. cerevisiae. Engineering and genetic selections revealed the required features of both subunits. Our data allow us to propose three minimal features required for ESCRT-III function - spiral formation, lateral association of the spirals through heteropolymerization, and binding to the AAA+ ATPase Vps4 for dynamic remodeling.

\section{Introduction:}

ESCRTs (endosomal sorting complexes required for transport) control a growing list of membrane remodeling events in cells (1). Among the different subcomplexes of ESCRTs, ESCRT-III is required in all processes, while the requirement of the other upstream complexes 0 , I and II is variable (2). Our understanding of the mechanisms of ESCRT-III assembly has 
increased substantially in the last decade (3-17). However, important questions remain. The specific role of each of the eight ESCRT-III subunits remain unclear. In eukaryotes, eight ESCRT-III proteins exist: Did2 (CHMP1), Vps2 (CHMP2), Vps24 (CHMP3), Snf7 (CHMP4), Vps60 (CHMP5), Vps20 (CHMP6), Chm7 (CHMP7) and Ist1 (IST1) (18, 19). Of these, Vps20,

34 Snf7, Vps24 and Vps2 have been studied in greater detail, because they are individually essential for MVB biogenesis in yeast, the earliest ascribed role for ESCRTs. These four proteins are the minimal subunits necessary to create intraluminal vesicles in the MVB pathway, as suggested by in vivo and in vitro analyses $(9,11,17,20)$. A recent study has further included Did2 and Ist1, providing additional insights into the sequential recruitment mechanism of ESCRT-III components (17). Toward a comprehensive understanding of each ESCRT-III subunit, studies in MVB biogenesis have provided important clues regarding their individual roles in membrane remodeling.

Vps24 and Vps2 are essential for MVB biogenesis $(3,21,22)$. Vps24 and Vps2 are also recruited cooperatively to membranes, requiring each one for the other's efficient recruitment $(9,10,21$, 23). Because of this reason, these two proteins have been analyzed together in previous ESCRT related work. Interestingly, in previous work, it was found that during HIV budding, while CHMP2 (the human ortholog of Vps2) is essential, CHMP3 (the human Vps24 ortholog) is not essential for HIV egress from cells (24). We hypothesized that there may be features of Vps24 and Vps2 that renders CHMP3 non-essential in some ESCRT-dependent processes. What are these essential features in these two proteins that make them indispensable for membrane remodeling? We set out to define those features in this work.

53 One of the most important features of Vps2 that has previously been described is the recruitment

54 of the AAA+ ATPase Vps4, which modifies ESCRT-III polymers(25, 26). Vps24 and Vps2 bind

55 to $\mathrm{Snf} 7$ and remodel Snf7 polymerization, changing the flat Snf7 spiral into 3D helices $(3,15$, 56 17, 23). We hypothesized that a single protein with features of Vps24 and Vps2 that can bind to

57 Snf7 but also recruit Vps4 may be sufficient for function. Here, through engineering approaches 58 we define a single protein that possesses such functions of both Vps24 and Vps2. These data 59 allow us to define the minimal essential properties of an ESCRT-III heteropolymer that are required for intraluminal vesicle formation. These include - a) spiral formation through a Snf7- 
61 like molecule, b) lateral association through a Vps24/Vps2 like molecule and c) the ability to

62 recruit the AAA+ATPase Vps4.

64 Results:

Overexpressing Vps2 can replace the function of Vps24 in MVB sorting

In our previous work (23), we observed that overexpressing VPS24 suppresses the defect of a snf7 allele $(\operatorname{snf7-D131K)}$ that encodes a Snf7 mutant with a lower affinity to Vps24. The overexpression, however, does not rescue other snf7 alleles that encode defective Snf7 homopolymers. We also observed that overexpression of Vps2 rescues the defect of $s n f 7-D 131 K$.

72 These data are consistent with the observations that Vps24 and Vps2 bind synergistically to Snf7

$73(9,10,21)$. Following these observations, we sought to test whether expressing a high level of

74 Vps2 also rescued the lack of Vps24 in cells, with the hypothesis that Vps2 may possess a lower

75 affinity binding surface for Snf7, which could be overcome with an increased availability of

$76 \quad$ Vps2 in the cytoplasm.

78 To test these hypotheses, we first utilized MVB cargo sorting assays (for cargoes Mup1 and Can1) in S. cerevisiae $(23,27)$. In a vps $24 \Delta$ strain overexpressing VPS2, we observed that Mup1-pHluorin is sorted at about $40 \%$ compared to that of the wild type, and that the canavanine sensitivity of

$81 v p s 24 \Delta$ is partially rescued (Figure 1A). We also noted that VPS2 overexpression rescues the

82 temperature sensitivity of vps $24 \Delta$ (Figure 1 - Fig. Supp 1A), suggesting that the increased

83 concentration of Vps2 could replace the cellular function of Vps24 beyond MVB cargo sorting.

85 With a tet-off regulatable operator, we next used doxycycline to titrate the expression level of Vps2. As a result, we determined that about 8-fold overexpression of Vps2 is necessary for

87 restoring Mup1 sorting (Figure 1 - Fig. Supp 1B-D). These data suggested that Vps2 contains features that can replace the function of Vps24 when present in higher concentrations in cells. 
93 To identify the features in Vps2 that could replace the function of Vps24, we utilized an

94 unbiased random-mutagenesis selection approach, as we have done previously (23, 28). Since

$95 v p s 24 \Delta$ is sensitive to the drug canavanine, we selected for $v p s 2$ mutants that conferred

96 canavanine resistance to vps $24 \Delta$ cells. We performed error-prone PCR and assembled a $v p s 2$

97 mutant library in a vps $24 \Delta$ strain. We next selected $v p s 2$ alleles using canavanine at a

98 concentration that the wild-type VPS2 does not grow (Figure 1B). From this selection, one of the

99 alleles (hereafter referred to as $\mathrm{Vps} 2^{\mathrm{RM}}$ ) strongly rescues the canavanine sensitivity of $v p s 24 \Delta$

100 (Figure 1C), and sorts Mup1-pHluorin to $45 \%$ of that of the wild-type (Figure 1C). Vps2 $2^{\mathrm{RM}}$

101 contains mutations in its promoter region, three missense mutations in helix-1 (N21K T28A

$102 \mathrm{E} 31 \mathrm{~K})$, and two missense mutations in helix-4 (S136N M146I).

103

104 The N-terminal mutations E21K T28A N31K (with the promoter mutations, hereafter called $105 \mathrm{Vps} 2 *$ ) are necessary and sufficient for the suppression effect. Interestingly, these mutants also 106 lie on the same surface of the alpha-1 helix, as a helical-wheel representation suggests (Fig. 1 107 Figure Supp. 3B). We found that while the individual mutations did not rescue vps $24 \Delta$ (Figure 1 108 - Fig. Supp. 2A), they collectively suppressed both the defect in canavanine sensitivity, and 109 Mup1 (sorting upto $\sim 40 \%$ ). Because the mutant we isolated also had promoter mutations, the 110 expression level of Vps2* is increased about 3-fold (Figure 1 - Fig. Supp 2C). The suppression 111 by $\mathrm{Vps} 2 *$ is not due simply to the overexpression effect however, since about 8-fold 112 overexpression of Vps 2 is required for only 20\% sorting of Mup1 to occur in a $v p s 24 \Delta$ strain 113 (Figure 1-Fig. Supp 1A-C).

115 To test whether Vps2* possesses features of both Vps24 and Vps2, we performed cargo sorting assays in $v p s 24 \Delta v p s 2 \Delta$. We observed that $v p s 2 *$ also suppresses $v p s 24 \Delta v p s 2 \Delta$ (Figure $2 \mathrm{~A}-\mathrm{B}$ ).

117 Therefore, a synergistic effect of the mutations in the N-terminal basic region of Vps2 helix-1

118 and its 3-fold overexpression provide the necessary functional features of Vps24 and Vps2.

120 One of the early-identified functions of Vps24 in yeast was as an adaptor for Vps2 to be 121 recruited to Snf7 polymers (20). In the absence of Vps24, Vps2 does not bind to Snf7 in coIP 
122 experiments (Figure $2 \mathrm{C}$ and as observed in (21),). This effect is rescued by the CMV promoter-

123 mediated overexpression of $\mathrm{Vps} 2$ or with $\mathrm{Vps} 2 *$

Vps2* binds to Snf7 at a lower expression level than the WT Vps2, providing further evidence that the N-terminal helix-1 mutations increase the affinity of Vps2 for Snf7, bypassing the need for Vps24 (Figure 2D). Although the overall feature of helix-1 region of Vps24 and Vps2 are similar (both basic helices), they vary in sequence composition (Figure 1 - Fig. Supp. 3A). Since the mutations occur in polar residues (Figure 1 - Fig. Supp. 3A-B), it is possible that these charge inversions increase the affinity of the basic patch of Vps2* to Snf7's acidic helix-4, consistent with our observations of Vps24 binding to Snf7 through an electrostatic interface (23). Simple overexpression of Vps2 probably rescues the defect of the lack of Vps 24 since the overall concentration of a lower-affinity Snf7-binding Vps2 molecule is increased in the cell.

\section{Binding to the AAA+ ATPase Vps4 is a critical feature of the Vps24-Vps2 module}

In contrast to Vps2 overexpression rescuing the defects of vps $24 \Delta$, the reverse doesn't occur Vps24 overexpression by $\sim 16$ fold did not rescue the defect of a $v p s 2 \Delta$ (Fig. 3). One of the critical features of $\mathrm{Vps} 2$ is the presence of the C-terminal MIM motif that has a higher affinity to the AAA+ ATPase Vps4 than other ESCRT-III proteins (25). While other ESCRT-III proteins also possess the MIM motifs, the Vps2 MIM has the strongest affinity for Vps4 in solution ( 20 $\mu \mathrm{M},(25))$. In addition, helix-5 of Vps2 has been identified as a second binding site of Vps4 with an affinity of $\sim 3 \mu \mathrm{M}(29-31)$. We therefore hypothesized that a Vps24 variant with Vps4 binding sites could possesses the properties of both Vps24 and Vps2. To test this, we replaced the Cterminus of Vps24 with that of Vps2 and assayed the functions of the chimeric protein.

We designed various chimeric constructs of Vps24/Vps2 under the control of their endogenous

148 promoters to first demarcate the regions that maintain function in a vps $24 \Delta$ strain (Figure 3 - Fig.

149 Supp1A). Consistent with the sequence analysis, replacing the MIM and helix5 of Vps24 with the homologous region of Vps2 kept the protein functional, but truncations beyond residue $\sim 152$ resulted in functional defects. In summary, the C-terminus (residues $\sim 152$ and beyond) of Vps 24 can be replaced with that of Vps2 and still retain function. 
154 We next tested these chimeras in $v p s 2 \Delta$. In contrast to vps $24 \Delta$ cells, we observed that they do not functionally restore the loss of Vps2 (Figure 3 - Fig. Supp 1B). To investigate whether they are dependent on protein expression levels, we then overexpressed these constructs with a CMV promoter that contain the N-terminus of Vps24 and helix-5 and MIM of Vps2. We found that they modestly suppress the defect of vps $2 \Delta$ to about $30 \%$ of wild-type (Figure 3 ). Therefore, directly recruiting Vps4 on to Vps24 partially bypasses the requirement of Vps2. We speculated that there are additional features in Vps24 that make it distinct from Vps2.

ESCRT-III subunits are soluble monomers in the cytoplasm but undergo structural rearrangements when assembled on membranes. We next investigated whether activating mutations in Vps24 that induce conformational changes renders it similar to Vps2. In our previous work (28), we designed a Snf7 mutant (hereafter referred to as Snf7***) that rescues the defects of vps204. Snf7*** includes a myristolyation site that recruits Snf7 to endosomes in the absence of upstream factors (ESCRT-I, ESCRT-II and Vps20) (28), as well as missense mutations (R52E Q90L N100I) that trigger Snf7 to adopt an elongated, open, and membranebound conformation $(2,22)$.

172 the interactions between helix-3 and helix-2 that would allow an extension of helices-2 and 3, 173 and therefore "activate" Vps24. As a result, we found that vps $24^{E 114 K}$ when overexpressed

174 robustly rescues the defect of $\operatorname{vps} 2 \Delta$ (Figure 3). Remarkably, this rescue becomes more 175 pronounced when vps $24^{E 114 K}$ carries the Vps4-binding motifs of Vps2 (Figure 3). Taken 176 together, our data suggest that a Vps24 variant capable of auto-activation and Vps4 recruitment 177 can function as Vps2.

Conformationally distinct Vps24 and Vps2 species replace one another back to alpha helices 1-2 $(8,19,32)$. During activation, the extension of helices 2-3 into an 
availability of an extended surface for self-assembly. Therefore, mutations that trigger conformational changes to an "open" state are able to readily form polymers in vivo and in vitro. Consistent with these ideas, Vps24 ${ }^{\mathrm{E} 114 \mathrm{~K}}$ in cell lysates forms higher-molecular weight species in glycerol-gradient experiments (Figure 4A). In in-vitro assays, while the wild-type Vps24 doesn't form polymers by itself or with $\mathrm{Vps} 2, \mathrm{Vps} 24^{\mathrm{E} 114 \mathrm{~K}}$ readily associates into linear filaments with Vps2 at concentrations of $5 \mu \mathrm{M}$ each (Figure 4B, Figure 4 - Fig. Supp. 1A). In comparison, previous experiments with $\mathrm{Vps} 24^{\mathrm{WT}}$ required 70 fold higher concentrations to observe similar linear polymers (33). With Snf7 and Vps2, both wild-type and the mutant Vps24 are able to form 3D spirals, which we previously described as the copolymeric structure of Snf7, Vps24 and Vps2 (Figure 4 - Fig. Supp. 1B). The increased ability to form polymers is consistent with the interpretation that E114K shifts the equilibrium of Vps24 to the open state, and that this open state mimics Vps2.

From sequence alignment analysis (Fig. 4C), we noticed low conservation between Vps24 and Vps 2 in the hinge between $\alpha 2$ and $\alpha 3$. Vps24 contains two helix-breaking asparagine residues in between these alpha helices (N99 and N103), while Vps2 lacks these helix-breaking residues (Figure 4C). We found that mutating the Asn to helix-stabilizing Ala (in addition to the Vps4 binding motifs) in Vps24 rescues the defect of $v p s 2 \Delta$, while the helix-breaking glycine does not rescue the defect (Figure 4B).

Therefore, it appears that the hinge region, that contains residues which affect the conformational flexibility of ESCRT-III proteins, in addition to the Vps4 binding sites, accounts for the majority

206 of the difference between Vps24 and Vps2. Consistent with this, when we overexpress a variant

207 that replaces the hinge region of Vps2 with that of Vps24, it suppresses vps $24 \Delta$ to $\sim 80 \%$ as

208 tested by Mup1 sorting, compared to $40 \%$ for that of the wild-type Vps2 (Figure 4D).

210 These data may suggest that Vps24 may not possess the ability to fully extend its helices-2 and 3

211 in the ESCRT-III copolymer. A recently published CryoEM structure showed that the Vps24

212 homopolymer consists of Vps24 protomers in a "semi-open" conformation (34) (see Fig. 4 - Fig.

213 Supp. 2 for direct comparison), in contrast to the fully extended and open Snf7 (8) and Did2 
214 (CHMP1) (16) polymers. It is possible that a mixture of different conformations allows for

215 efficient Vps24-Vps2 assembly, which has a higher affinity to the Snf7 polymer.

217 Collectively, these data suggest that while Vps24 and Vps2 are similar proteins. Laterally

218 interacting with Snf7, inducing the formation of an ESCRT-III super-helix, and recruiting Vps4

219 are three features for the Vps24/Vps2 module.

\section{"Accessory" ESCRT-III genes promote intralumenal vesicle formation}

In Saccharomyces cerevisiae, there are eight ESCRT-III genes. One of the defining features of

224 these ESCRT-III proteins is the N-terminal alpha-helical bundle, which is sometimes referred to 225 as the ESCRT-III domain. The other defining feature is the C-terminal flexible region that 226 contains at least one MIM, that binds to the MIT domain of Vps4. The N-terminus of the 227 ESCRT-III domain are similar in sequence and structure. However, the specific functions of all these ESCRT-III proteins remains unclear.

To quantitatively assess the relative contributions among the ESCRT proteins, we assayed for

231 Mup1-pHluorin sorting in each gene deletion (Figure 5- Fig. Supp 1). We also performed

232 canavanine sensitivity assays (Fig. 5 - Fig. Supp 1 B) with the same mutants. We observed that

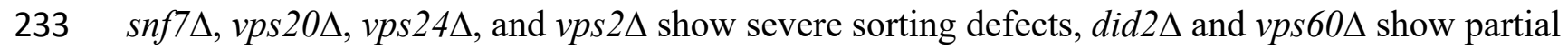
234 sorting defects, and ist $1 \Delta$ and $\operatorname{chm} 7 \Delta$ show no defect. These data are consistent with previous 235 findings with a different cargo (CPS) (18), and from in-vitro assays $(11,17)$, which suggest that 236 Snf7, Vps20, Vps24 and Vps2 are the minimal contributors in MVB formation.

238 These differences in function during MVB formation occur despite similarity in structure and 239 sequence between these ESCRT-III proteins. Inspired by the observation that VPS2 240 overexpression suppresses vps $24 \Delta$, we investigated whether overexpressing other ESCRT-III 241 genes could suppress the deletions of a different ESCRT-III gene. We used the CMV promoter to 242 overexpress each of the ESCRT-III genes: Snf7 is overexpressed by $\sim 5$ fold, and Vps24 and 243 Vps2 by $\sim 16$ fold. Most of the overexpression constructs didn't rescue the defect of the other 
244 ESCRT-III deletions, except in two cases. As described above, Vps2 overexpression rescued the

245 defect of $v p s 24 \Delta$, and also partially rescued the defect of a $\operatorname{did} 2 \Delta$.

247 Evolutionary analyses have grouped ESCRT-III into two groups: Snf7/Vps20/Vps60, and

248 Vps24/Vps2/Did2, (Fig. 6A) (35, 36). Vps20 nucleates formation of Snf7 spirals, and

$249 \mathrm{Vps} 24 / \mathrm{Vps} 2$ induce bundling and helix formation of spirals (23). In in-vitro assays with lipid

250 monolayers, we found that Did2 forms tube-like helices (Fig. 6B, and as previously shown in (7,

251 16) for mammalian Did2 named CHMP3). However, Vps60 lacks the ability to form long

252 helices/tubes, and preferentially forms spiral-like structures, reminiscent of Snf7 (Fig. 6B).

253 Consistent with Vps60 mimicking Snf7 structurally, the N-terminal region of Snf7 fused to the

254 C-terminal region of Vps60 rescues the defects of vps60د (Fig. 6C). Vps60-GFP is localized to

255 endosomal and vacuolar membranes with a hint of plasma membrane signal (Fig. 6 - Figure

256 Supp. 1). This localization is primarily cytosolic in $v p s 20 \Delta$, snf7 $\Delta$ or $v p s 2 \Delta$, and unchanged in

$257 \operatorname{did} 2 \Delta$ (Fig. 6 - Figure Supp. 1).

In summary, among Vps20/Snf7/Vps20, Snf7 serves as the main scaffold which can be engineered to substitute for Vps20 (28) or Vps60 (Fig. 6A). Among Vps24/Vps2/Did2,

261 modifications within Vps24/Vps2 can functionally replace each other; Did2 resembles

262 Vps24/Vps2 as it readily forms three-dimensional helices, and did2 $\Delta$ can be partially suppressed

263 by Vps2 overexpression. Our data suggest that although ESCRT-III subunits have evolved for

264 divergent roles in ordered assembly, rational modifications in ESCRT-III subunits can allow one 265 to consolidate the functions of two ESCRT-III proteins into one ESCRT-III protein.

\section{Discussion:}

In this work we have utilized rational design and unbiased mutagenesis to understand the design

271 ESCRT-III subunits Vps24 and Vps2. First, we find that overexpression of Vps2, by $\sim$-fold and

272 above, can rescue vps $24 \Delta$ in yeast. Second, point mutations in the helix-1 region of Vps 2 can

273 also rescue vps24A. These Vps2 mutants also bind to Snf7 in-vivo even in the absence of Vps24.

274 Third, overexpression and inclusion of higher-affinity AAA + ATPase Vps4 binding regions on 
275 Vps24 can rescue the absence of Vps2. Fourth, mutations that induce conformational changes in

276 Vps24 and Vps2 also rescue each other's function. These data indicate a strong similarity in

277 between these two ESCRT-III subunits.

278

279 Despite the observed similarity between Vps24 and Vps2, our data also suggest some

280 differences: they may exist in different conformations (Fig. 7A), and that Vps2 consists of a

281 higher-affinity Vps4 binding site (Fig. 7B). In addition, Vps24/Vps2 induce lateral association

282 and bundling $(10,17,23)$, along with helicity of the spirals, which could be an important

283 parameter for ESCRT-III function for MVB biogenesis.

Following these observations, we propose the following three critical aspects of an ESCRT-III

minimal module: (1) a core spiral forming unit (e.g., Snf7), (2) a lateral bundling unit (e.g., Vps24 and Vps2), and (3) an ability to recruit a disassembly machinery (e.g., the AAA+ ATPase Vps4) (Figure 7C).

In addition to the rescue of function phenotypes with Vps24/Vps2, we also find that did $2 \Delta$ can be rescued partially with the overexpression of Vps2. Similarly, swapping the C-termini of Snf7 with

292 that of Vps60 can replace the function of vps604. We previously showed that point mutations in 293 Snf7 can rescue the absence of Vps20 (28). These data imply, as predicted by evolutionary 294 analyses $(35,37)$, that Vps20-Snf7-Vps60 are more similar to one another, and Vps2-Vps24-Did2 295 are more alike one another, given the rescue of vps $20 \Delta$ or $v p s 60$ with Snf7 alleles, and vps $24 \Delta$ and did $2 \Delta$ with $\mathrm{Vps} 2$ alleles.

In vitro reconstitutions suggest that $\mathrm{Snf} 7, \mathrm{Vps} 24$, and Vps2 are essential for membrane budding, and Vps4 for vesicle scission $(11,17)$. Vps4-mediated turnover of a laterally-associating and helix-inducing polymer of Vps24-Vps2 would constrict the Snf7 scaffold to a fission-competent structure, as predicted by simulations (38) and as recently proposed to occur in archaeal cell division (39). While Did2 and Ist1 are not essential for intraluminal vesicle formation in vitro and in vivo, they have regulatory roles that are controlled by sequential polymerization dynamics 304 through Vps4 (17). So far Vps60 has not been included in in-vitro analyses and there have been 
membranes, Vps2 (and likely Vps24) are required. Therefore, Vps60 may be recruited in later stages of polymer formation, in a sequential fashion to the core scaffold, as Did2 does (17). Vps60 has been shown to interact with Vta1(40-42), and Vta1 is known to be an activator of Vps4 $(40,43,44)$. Therefore, it is possible that Vps60 is involved in further activating Vps4 function in the later stages of polymer dynamics.

ESCRT-III proteins are integral to all ESCRT-related functions in cells. However, the mechanisms of the specific roles of each ESCRT-III proteins have remained unclear. Based on our work on Vps24 and Vps2 described here and our previous studies on Snf7 and Vps20 (28), the molecular features of ESCRT-III subunits should enable future work on rational design of minimal ESCRT-III subunit(s) possessing all properties necessary for intraluminal vesicle

317 formation. Further in vivo analyses and in vitro reconstitution are required to test whether this minimal ESCRT-III subunit(s) can be created that include the aforementioned features. Some archaeal species consist of only two ESCRT-III proteins, which must possess the minimal properties of ESCRT-III necessary for function (36). With the principles learned from our work and from recent studies on ESCRT-III, it will be interesting to study what biochemical features the ancient archaeal ESCRT-III subunits consist, and what additional features were acquired as eukaryotic organelles evolved.

Our earlier understanding of Vps24 and Vps2 suggested that they bound cooperatively to Snf7,

326 but that these were independent proteins with independent and specific functions for MVB 327 sorting. Our data in this study suggest that minor modifications to either one can replace the 328 function of another. These data provide an explanation for why in certain biological processes 329 CHMP3 (mammalian Vps24) may play a minor role (such as in HIV budding), as the isoforms of CHMP2 (Vps2) may already possess the ability to form lateral interactions, and also an ability to recruit the AAA+ ATPase Vps4. The relative contribution of the different ESCRT-III proteins

332 for other ESCRT-dependent processes have not been quantified to the same extent. Further 333 analysis of site-specific ESCRT-III function could allow us to achieve targeted cellular 334 manipulation of ESCRT-dependent processes, understanding of the evolution of these 335 membrane-remodeling polymers and how they contribute to organelle biogenesis. 


\section{Acknowledgments}

338 We thank David Teis for the gift of anti-Vps2 antibody. We thank all members of the Emr lab

339 for discussions. Work in the Emr lab is supported by a Cornell University Research Grant

340 CU3704. Sudeep Banjade is an HHMI fellow of the Damon Runyon Cancer Research

341 Foundation (DRG-2273-16). We are also grateful to the Damon Runyon Cancer Research

342 Foundation for an extension of the fellowship to support our work during the COVID-19

343 pandemic delays. Shaogeng Tang is a Merck fellow of the Damon Runyon Cancer Research

344 Foundation (DRG-2301-17) on a different project.

Materials and Methods

\section{Strains, Plasmids and Reagents}

349 Strains, plasmids and reagents are described in Table 1. Strains previously used were from (3, $35018,21-23)$, and also referenced in the table.

\section{Random mutagenesis selection}

353 Error prone PCR was used to generate random mutations in the plasmid harboring the S.

354 cerevisiae Vps2 gene. The primers used for this PCR bind the 5' UTR and the 3' UTR regions of

$355 \mathrm{Vps} 2$. The PCR fragment was transformed into the vps $24 \Delta$ strain in the presence of a linearized

356 Vps2 plasmid by digesting with HindIII and NarI enzymes. The transformants were plated onto

357 plates containing $0.5 \mu \mathrm{g} / \mathrm{mL}$ canavanine, and then replica plated into $4 \mu \mathrm{g} / \mathrm{mL}$ plates. Plasmids

358 were rescued from these colonies that grew on canavanine and then re-transformed into the $359 v p s 24 \Delta$ strain to confirm the suppression of $v p s 24 \Delta$. Confirmation of $v p s 24 \Delta$ suppression was

360 done by testing Mup1-pHluorin sorting ability (see below) of the mutants.

\section{Canavanine Spot Plates}

363 Mid-log cells were serially were diluted to an $\mathrm{OD}_{600}$ of 0.1 . They were then diluted 10-fold 364 serially, and spot plated in plates containing various concentrations of canavanine. Images of the 365 plates were taken at 3 and 5 days. 
Strains harbring Mup1-pHluorin were used to assay endocytosis of this cargo upon methionine addition. Assays were performed as described before (28). Briefly, mid-log cells in the presence of synthetic drop-out media were treated with $20 \mu \mathrm{g} / \mathrm{mL}$ L-methionine for 90 minutes and assayed for quenching of pHluorin. Over time as Mup1-pHluorin traffics to the vacuole,

372 fluorescence decreases due to quenching of the $\mathrm{pH}$ sensitive pHluorin. Experiments were

373 performed at room temperature and analyses were done on a C6 Accuri flow cytometer from BD

374 Biosciences.

Immunoblots after methionine treatment were performed to analyze free pHlourin, upon degradation of Mup1, as described (23). Blots were performed using primary antibody against GFP from Torrey Pines. Imaging of the western blots was performed using an Odyssey CLx imaging system and analyzed using the Image Studio Lite 4.0.21 software (LI-COR Biosciences).

\section{Doxycycline-mediated Shutdown of the Tet-off Operator}

Plasmids (pCM189) used in this study that under the tet-off operator have a CMV promoter and can be regulated by doxycycline titration. For titration experiments, cells were diluted to an OD600 of 0.01 . Doxycyline was added at a concentration of $0.25 \mu \mathrm{g} / \mathrm{mL}$ and serially diluted 2fold over eight times. Cultures were grown until an OD600 of 0.5, and then treated with methionine for Mup1-pHlourin sorting assays or used for co-immunoprecipitation.

\section{Protein Purification}

Vps24, Snf $7^{\mathrm{R} 52 \mathrm{E}}$ and Vps2 constructs used in this study were purified as described before (23). A combination of affinity (Cobalt Talon resin) and size-exclusion chromatography (SD200increase,

392 GE) were used to purify the proteins. The final buffer under which the proteins are stored was 25 $393 \mathrm{mM}$ Hepes $\mathrm{pH}$ 7.5, $150 \mathrm{mM} \mathrm{NaCl}$ and $2 \mathrm{mM} \beta$-ME. His6-tagged Vps60 and Did2 were purified through cobalt and anion exchange chromatography.

\section{Electron Microscopy}

397 Lipid monolayers were prepared with a mixture of $60 \%$ POPC, $30 \%$ POPS and 10\% PI3P in

398 chloroform. Carbon-coated electron microscope grids were used to make monolayers and 
incubate with proteins, as described before(27). Grids were stained with $2 \%$ ammonium molybdate and imaged on an FEI Morgagni 268 TEM.

\section{Glycerol Gradient}

403 For Vps24 and Vps24 E114K glycerol gradients, vps244 was transformed with pCM189 Vps24

404 or pCM189 Vps24 E114K. 30 ODs of cells expressing these constructs were harvested in PBS

405 buffer. Lysis was performed with PBS buffer, 10\% glycerol, 1 mM DTT, Roche protease

406 cocktail and 0.5\% Tween-20. Gradient Master 108 from Biocomp was used to make glycerol

407 gradients of 10 to $40 \%$. Centrifugation was performed at $100,000 \mathrm{xg}$ for 4 hours at $4^{\circ} \mathrm{C} .1 \mathrm{~mL}$

408 fractions were collected from the solutions, TCA precipitated and immunoblotted.

Fluorescence microscopy

$4111 \mathrm{~mL}$ of mid-log cells was harvested and resuspended in $25 \mu \mathrm{L}$ water. Imaging was performed on 412 a Deltavision Elite system with an Olympus IX-71 inverted microscope, using a 100X/1.4 NA oil 413 objective. Image extraction and analysis were performed using the FiJi software.

\section{Sequence and structural analyses}

416 Mafft(45) and Jalview (46) were used to analyze sequences. Heliquest was used for helical wheel 417 analysis(47). Structural models were made using UCSF Chimera(48).

\section{Co-immuoprecipitation}

42030 ODs of mid-log cells were harvested and washed with cold MilliQ $\mathrm{H}_{2} \mathrm{O}$, and resuspended in 1

$421 \mathrm{~mL}$ phosphate-saline buffer (PBS), 10\% glycerol, $1 \mathrm{mM}$ DTT and $1 \mathrm{mM}$ EDTA, including

422 protease inhibitor cocktail from Roche. Lysis was performed by bead-beating (Zirconia-Silicon

423 beads) twice for $30 \mathrm{~s}$, with 30s intervals on ice. Lysate was treated with 1\% Triton X-100 and

424 rotated for 20 minutes. Lysate was cleared by centrifugation at $16,000 \mathrm{xg}$ at $4{ }^{\circ} \mathrm{C}$. The supernatant

425 was treated with protein $\mathrm{G}$ beads (Dynabeads) for $30 \mathrm{~min}$ at $4^{\circ} \mathrm{C}$ to remove nonspecific binding.

426 The magnetic beads used for this assay were allowed to settle with a magnetic Eppendorf-tube

427 rack, and the supernatant was applied with 1/250 v/v of anti-Snf7 antibody. After 1-hour

428 incubation at $4^{\circ} \mathrm{C}$, the beads were washed twice with $20 \mathrm{X}$ fold bead volume of the lysis buffer.

429 Proteins were eluted by incubating the beads at $65^{\circ} \mathrm{C}$ for 10 minutes in sample buffer $(150 \mathrm{mM}$ 
Tris-Cl, pH 6.8, $8 \mathrm{M}$ urea, 10\% SDS, 24\% glycerol, 10\% v/v ME, and bromophenol blue).

431 Anti-Snf7 and anti-Vps2 antibodies were used to probe for eluted proteins through Western

432 blots.

\section{References}

434 1. M. Vietri, M. Radulovic, H. Stenmark, The many functions of ESCRTs. Nat. Rev. Mol. Cell Biol. 21, 25-42 (2020).

2. W. M. Henne, H. Stenmark, S. D. Emr, Molecular mechanisms of the membrane sculpting ESCRT pathway. Cold Spring Harb Perspect Biol. 5 (2013), doi:10.1101/cshperspect.a016766.

3. W. M. Henne, N. J. Buchkovich, Y. Zhao, S. D. Emr, The endosomal sorting complex ESCRT-II mediates the assembly and architecture of ESCRT-III helices. Cell. 151, 356371 (2012).

4. Q.-T. Shen, A. L. Schuh, Y. Zheng, K. Quinney, L. Wang, M. Hanna, J. C. Mitchell, M. S.

7. J. McCullough, A. K. Clippinger, N. Talledge, M. L. Skowyra, M. G. Saunders, T. V.

5. A. G. Cashikar, S. Shim, R. Roth, M. R. Maldazys, J. E. Heuser, P. I. Hanson, Structure of mechanisms governing ESCRT-III spiral filament assembly. J. Cell Biol. 206, 763-777 (2014).

6. N. Chiaruttini, L. Redondo-Morata, A. Colom, F. Humbert, M. Lenz, S. Scheuring, A. Roux, Relaxation of Loaded ESCRT-III Spiral Springs Drives Membrane Deformation. Cell. 163, 866-879 (2015). Naismith, L. A. Colf, P. Afonine, C. Arthur, W. I. Sundquist, P. I. Hanson, A. Frost, Structure and membrane remodeling activity of ESCRT-III helical polymers. Science. 350, 1548-1551 (2015).

8. S. Tang, W. M. Henne, P. P. Borbat, N. J. Buchkovich, J. H. Freed, Y. Mao, J. C. Fromme, S. D. Emr, Structural basis for activation, assembly and membrane binding of ESCRT-III Snf7 filaments. Elife. 4 (2015), doi:10.7554/eLife. 12548.

9. M. A. Y. Adell, S. M. Migliano, S. Upadhyayula, Y. S. Bykov, S. Sprenger, M. Pakdel, G. F. Vogel, G. Jih, W. Skillern, R. Behrouzi, M. Babst, O. Schmidt, M. W. Hess, J. A. Briggs, T. Kirchhausen, D. Teis, Recruitment dynamics of ESCRT-III and Vps4 to endosomes and implications for reverse membrane budding. Elife. 6 (2017), doi:10.7554/eLife.31652.

10. B. E. Mierzwa, N. Chiaruttini, L. Redondo-Morata, J. M. von Filseck, J. König, J. Larios, I. Poser, T. Müller-Reichert, S. Scheuring, A. Roux, D. W. Gerlich, Dynamic subunit turnover in ESCRT-III assemblies is regulated by Vps4 to mediate membrane remodelling during cytokinesis. Nat. Cell Biol. 19, 787-798 (2017). 
11. J. Schöneberg, M. R. Pavlin, S. Yan, M. Righini, I.-H. Lee, L.-A. Carlson, A. H. Bahrami, D. H. Goldman, X. Ren, G. Hummer, C. Bustamante, J. H. Hurley, ATP-dependent force generation and membrane scission by ESCRT-III and Vps4. Science. 362, 1423-1428 (2018).

12. I. Goliand, S. Adar-Levor, I. Segal, D. Nachmias, T. Dadosh, M. M. Kozlov, N. Elia, Resolving ESCRT-III Spirals at the Intercellular Bridge of Dividing Cells Using 3D STORM. Cell Rep. 24, 1756-1764 (2018).

13. S. Maity, C. Caillat, N. Miguet, G. Sulbaran, G. Effantin, G. Schoehn, W. H. Roos, W. Weissenhorn, VPS4 triggers constriction and cleavage of ESCRT-III helical filaments. Sci $A d v$. 5, eaau7198 (2019).

14. A. Bertin, N. de Franceschi, E. de la Mora, S. Maity, M. Alqabandi, N. Miguet, A. di Cicco, W. H. Roos, S. Mangenot, W. Weissenhorn, P. Bassereau, Human ESCRT-III polymers assemble on positively curved membranes and induce helical membrane tube formation. Nat Commun. 11, 2663 (2020).

15. J. Moser von Filseck, L. Barberi, N. Talledge, I. E. Johnson, A. Frost, M. Lenz, A. Roux, Anisotropic ESCRT-III architecture governs helical membrane tube formation. Nat Commun. 11, 1516 (2020).

16. H. C. Nguyen, N. Talledge, J. McCullough, A. Sharma, F. R. Moss, J. H. Iwasa, M. D. Vershinin, W. I. Sundquist, A. Frost, Membrane constriction and thinning by sequential ESCRT-III polymerization. Nat. Struct. Mol. Biol. 27, 392-399 (2020).

17. A.-K. Pfitzner, V. Mercier, X. Jiang, J. Moser von Filseck, B. Baum, A. Šarić, A. Roux, An ESCRT-III Polymerization Sequence Drives Membrane Deformation and Fission. Cell (2020), doi:10.1016/j.cell.2020.07.021.

18. S. M. Rue, S. Mattei, S. Saksena, S. D. Emr, Novel Ist1-Did2 complex functions at a late step in multivesicular body sorting. Mol. Biol. Cell. 19, 475-484 (2008).

19. J. McCullough, A. Frost, W. I. Sundquist, Structures, Functions, and Dynamics of ESCRTIII/Vps4 Membrane Remodeling and Fission Complexes. Annu. Rev. Cell Dev. Biol. 34, 85-109 (2018).

20. D. Teis, S. Saksena, S. D. Emr, Ordered assembly of the ESCRT-III complex on endosomes is required to sequester cargo during MVB formation. Dev. Cell. 15, 578-589 (2008).

21. M. Babst, D. J. Katzmann, E. J. Estepa-Sabal, T. Meerloo, S. D. Emr, Escrt-III: an endosome-associated heterooligomeric protein complex required for mvb sorting. Dev. Cell. 3, 271-282 (2002).

22. N. J. Buchkovich, W. M. Henne, S. Tang, S. D. Emr, Essential N-terminal insertion motif anchors the ESCRT-III filament during MVB vesicle formation. Dev. Cell. 27, 201-214 (2013). 
23. S. Banjade, S. Tang, Y. H. Shah, S. D. Emr, Electrostatic lateral interactions drive ESCRTIII heteropolymer assembly. Elife. 8 (2019), doi:10.7554/eLife.46207.

24. E. Morita, V. Sandrin, J. McCullough, A. Katsuyama, I. Baci Hamilton, W. I. Sundquist, ESCRT-III protein requirements for HIV-1 budding. Cell Host Microbe. 9, 235-242 (2011).

25. T. Obita, S. Saksena, S. Ghazi-Tabatabai, D. J. Gill, O. Perisic, S. D. Emr, R. L. Williams, Structural basis for selective recognition of ESCRT-III by the AAA ATPase Vps4. Nature. 449, 735-739 (2007).

26. M. A. Y. Adell, G. F. Vogel, M. Pakdel, M. Müller, H. Lindner, M. W. Hess, D. Teis, Coordinated binding of Vps4 to ESCRT-III drives membrane neck constriction during MVB vesicle formation. J Cell Biol. 205, 33-49 (2014).

27. S. Banjade, S. Tang, S. D. Emr, Genetic and Biochemical Analyses of Yeast ESCRT. Methods Mol. Biol. 1998, 105-116 (2019).

28. S. Tang, N. J. Buchkovich, W. M. Henne, S. Banjade, Y. J. Kim, S. D. Emr, ESCRT-III activation by parallel action of ESCRT-I/II and ESCRT-0/Bro1 during MVB biogenesis. Elife. 5 (2016), doi:10.7554/eLife.15507.

29. H. Han, N. Monroe, J. Votteler, B. Shakya, W. I. Sundquist, C. P. Hill, Binding of Substrates to the Central Pore of the Vps4 ATPase Is Autoinhibited by the Microtubule Interacting and Trafficking (MIT) Domain and Activated by MIT Interacting Motifs (MIMs). J. Biol. Chem. 290, 13490-13499 (2015).

30. H. Han, N. Monroe, W. I. Sundquist, P. S. Shen, C. P. Hill, The AAA ATPase Vps4 binds ESCRT-III substrates through a repeating array of dipeptide-binding pockets. Elife. 6 (2017), doi:10.7554/eLife.31324.

31. H. Han, J. M. Fulcher, V. P. Dandey, J. H. Iwasa, W. I. Sundquist, M. S. Kay, P. S. Shen, C. P. Hill, Structure of Vps4 with circular peptides and implications for translocation of two polypeptide chains by AAA+ ATPases. Elife. 8 (2019), doi:10.7554/eLife.44071.

32. M. Bajorek, H. L. Schubert, J. McCullough, C. Langelier, D. M. Eckert, W.-M. B. Stubblefield, N. T. Uter, D. G. Myszka, C. P. Hill, W. I. Sundquist, Structural basis for ESCRT-III protein autoinhibition. Nat. Struct. Mol. Biol. 16, 754-762 (2009).

33. S. Ghazi-Tabatabai, S. Saksena, J. M. Short, A. V. Pobbati, D. B. Veprintsev, R. A. Crowther, S. D. Emr, E. H. Egelman, R. L. Williams, Structure and disassembly of filaments formed by the ESCRT-III subunit Vps24. Structure. 16, 1345-1356 (2008).

34. S. T. Huber, S. Mostafavi, S. A. Mortensen, C. Sachse, Structure and assembly of ESCRTIII helical Vps24 filaments. Sci Adv. 6, eaba4897 (2020).

35. K. F. Leung, J. B. Dacks, M. C. Field, Evolution of the multivesicular body ESCRT machinery; retention across the eukaryotic lineage. Traffic. 9, 1698-1716 (2008). 
36. Y. Caspi, C. Dekker, Dividing the Archaeal Way: The Ancient Cdv Cell-Division Machinery. Front Microbiol. 9, 174 (2018).

37. A. Spang, J. H. Saw, S. L. Jørgensen, K. Zaremba-Niedzwiedzka, J. Martijn, A. E. Lind, R. van Eijk, C. Schleper, L. Guy, T. J. G. Ettema, Complex archaea that bridge the gap between prokaryotes and eukaryotes. Nature. 521, 173-179 (2015).

38. L. Harker-Kirschneck, B. Baum, A. E. Šarić, Changes in ESCRT-III filament geometry drive membrane remodelling and fission in silico. BMC Biol. 17, 82 (2019).

39. G. Tarrason Risa, F. Hurtig, S. Bray, A. E. Hafner, L. Harker-Kirschneck, P. Faull, C. Davis, D. Papatziamou, D. R. Mutavchiev, C. Fan, L. Meneguello, A. Arashiro Pulschen, G. Dey, S. Culley, M. Kilkenny, D. P. Souza, L. Pellegrini, R. A. M. de Bruin, R. Henriques, A. P. Snijders, A. Šarić, A.-C. Lindås, N. P. Robinson, B. Baum, The proteasome controls ESCRT-III-mediated cell division in an archaeon. Science. 369 (2020), doi:10.1126/science.aaz2532.

40. Z. Yang, C. Vild, J. Ju, X. Zhang, J. Liu, J. Shen, B. Zhao, W. Lan, F. Gong, M. Liu, C. Cao, Z. Xu, Structural Basis of Molecular Recognition between ESCRT-III-like Protein Vps60 and AAA-ATPase Regulator Vta1 in the Multivesicular Body Pathway $\$$. Biol Chem. 287, 43899-43908 (2012).

41. I. F. Azmi, B. A. Davies, J. Xiao, M. Babst, Z. Xu, D. J. Katzmann, ESCRT-III Family Members Stimulate Vps4 ATPase Activity Directly or via Vta1. Developmental Cell. 14, 50-61 (2008).

42. D. P. Nickerson, M. West, R. Henry, G. Odorizzi, Regulators of Vps4 ATPase activity at endosomes differentially influence the size and rate of formation of intralumenal vesicles. Mol Biol Cell. 21, 1023-1032 (2010).

43. A. P. Norgan, B. A. Davies, I. F. Azmi, A. S. Schroeder, J. A. Payne, G. M. Lynch, Z. Xu, D. J. Katzmann, Relief of Autoinhibition Enhances Vta1 Activation of Vps4 via the Vps4 Stimulatory Element. J Biol Chem. 288, 26147-26156 (2013).

44. N. Monroe, H. Han, P. S. Shen, W. I. Sundquist, C. P. Hill, Structural basis of protein translocation by the Vps4-Vta1 AAA ATPase. eLife. 6, e24487 (2017).

45. K. Katoh, K. Misawa, K. Kuma, T. Miyata, MAFFT: a novel method for rapid multiple sequence alignment based on fast Fourier transform. Nucleic Acids Res. 30, 3059-3066 (2002).

46. M. Clamp, J. Cuff, S. M. Searle, G. J. Barton, The Jalview Java alignment editor. Bioinformatics. 20, 426-427 (2004).

47. R. Gautier, D. Douguet, B. Antonny, G. Drin, HELIQUEST: a web server to screen sequences with specific alpha-helical properties. Bioinformatics. 24, 2101-2102 (2008). 
bioRxiv preprint doi: https://doi.org/10.1101/2020.12.28.424573; this version posted December 30, 2020. The copyright holder for this preprint (which was not certified by peer review) is the author/funder, who has granted bioRxiv a license to display the preprint in perpetuity. It is made available under aCC-BY-NC-ND 4.0 International license.

574

575

576

577
48. E. F. Pettersen, T. D. Goddard, C. C. Huang, G. S. Couch, D. M. Greenblatt, E. C. Meng, T. E. Ferrin, UCSF Chimera--a visualization system for exploratory research and analysis. $J$ Comput Chem. 25, 1605-1612 (2004). 


\section{Figure 1}
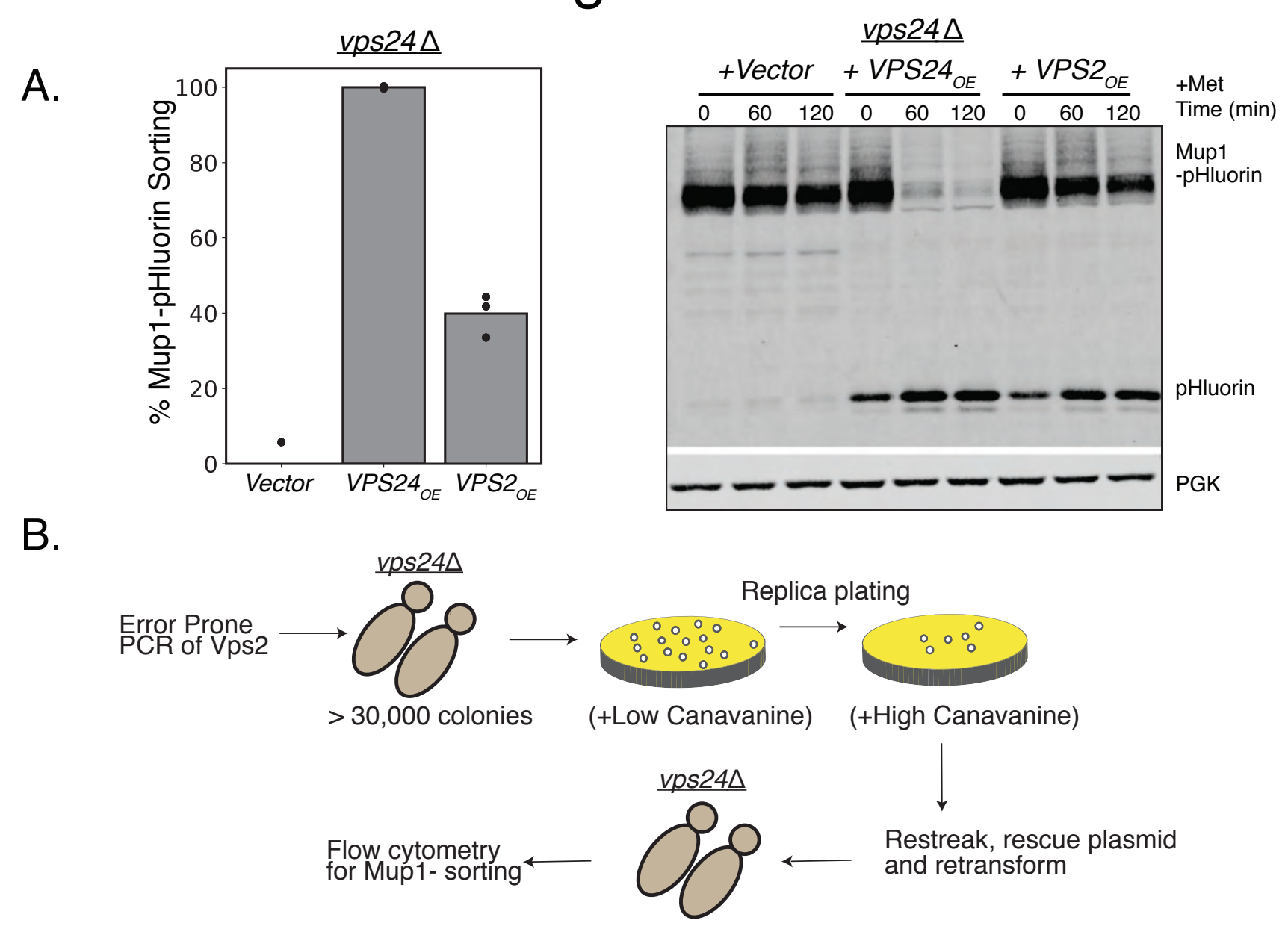

C.

vps24

vps24 $\triangle$

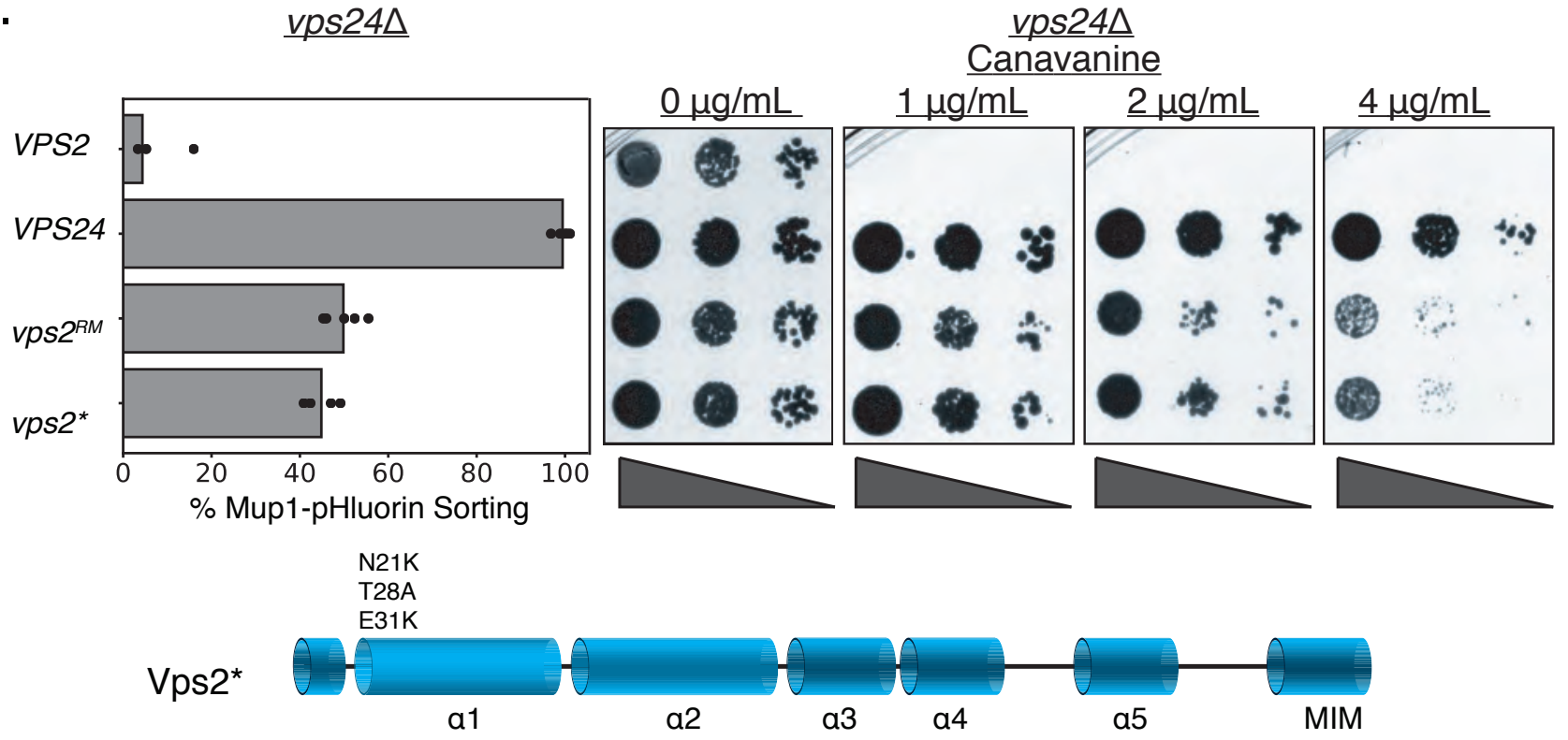

Figure 1. Minor modifications in Vps2 can replace the function of Vps24. A) Overexpression of Vps2 can rescue the defect of $v p s 24 \Delta$ for Mup1 sorting. Image on the left represents Mup-pHluorin sorting through a flow-cytometry assay and the image on the right represents an immunoblot for pHluorin upon methionine addition. Overexpression (OE) was achieved through a CMV-promoter and Tet-operator containing plasmid. B)Flow-chart of the random mutagenesis approach. C) Top figure shows the flow cytometry and canavanine sensitivity assays with the mutant Vps2 that can rescue the sorting defects of $v p s 24 \Delta$. Bottom figure shows the domains of Vps2 highlighting the mutations found in the selection. 


\section{Figure 1 - Figure Supplement 1}
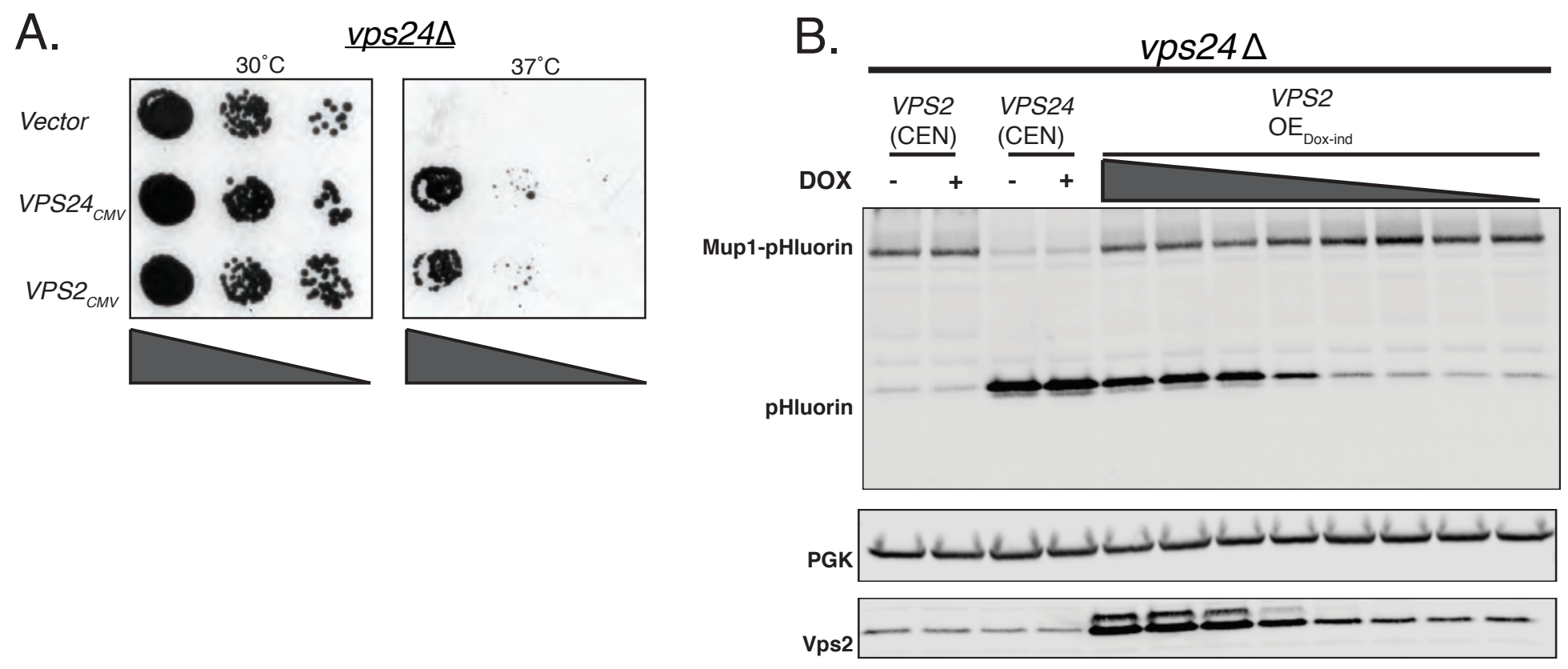

C.

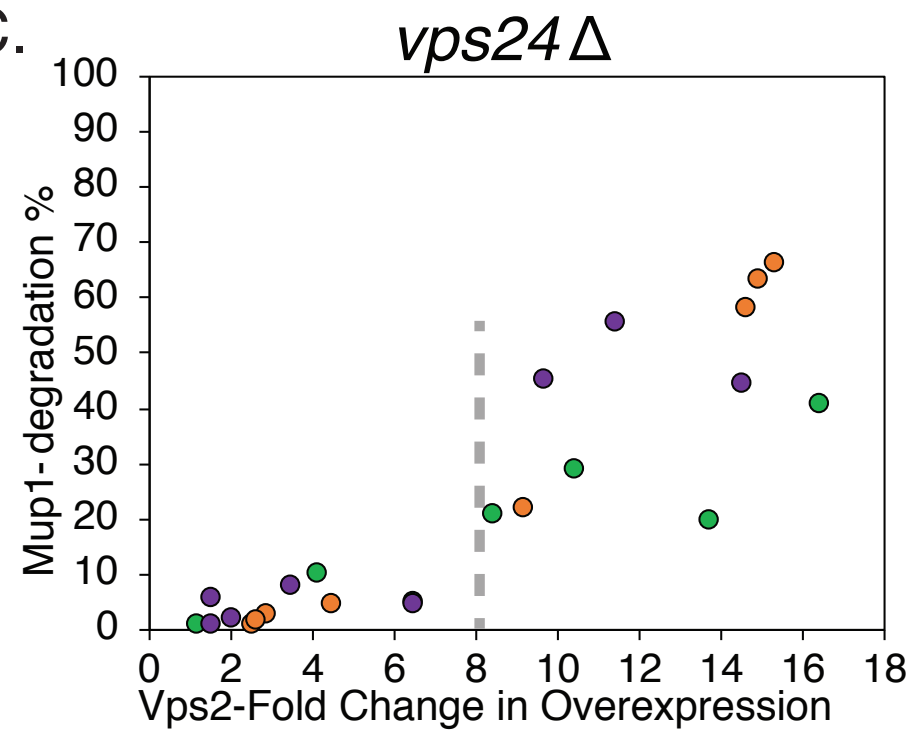

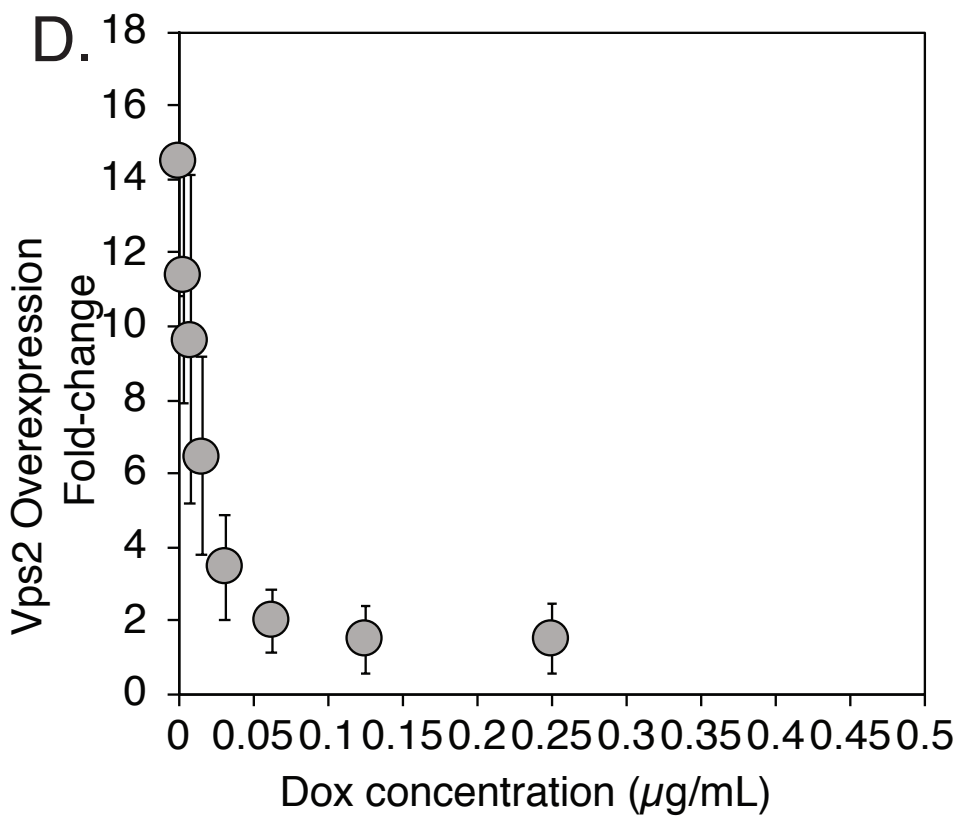

Figure 1 - Fig. Supp. 1. Vps2 overexpression can rescue the defect of vps24A. A)Vps2 overexpression with a CMV-promoter/Tet-operator rescues the temperature sensitivity defect of $v p s 24 \Delta$. B) Immunoblot of pHLuorin showing the cleavage of Mup1-pHluorin after 90 minutes of methionine addition. Vps2 was expressed either in a single-copy centromeric (CEN) plasmid or under a doxycycline-inducible CMV promoter. Expression of Vps2 was controlled by titrating the concentration of doxycycline. C) Mup1-sorting characterization with changes in Vps2 expression level. The different colors represent different set titration experiments. D) Plot showing the control of expression levels with doxycycline titration. 


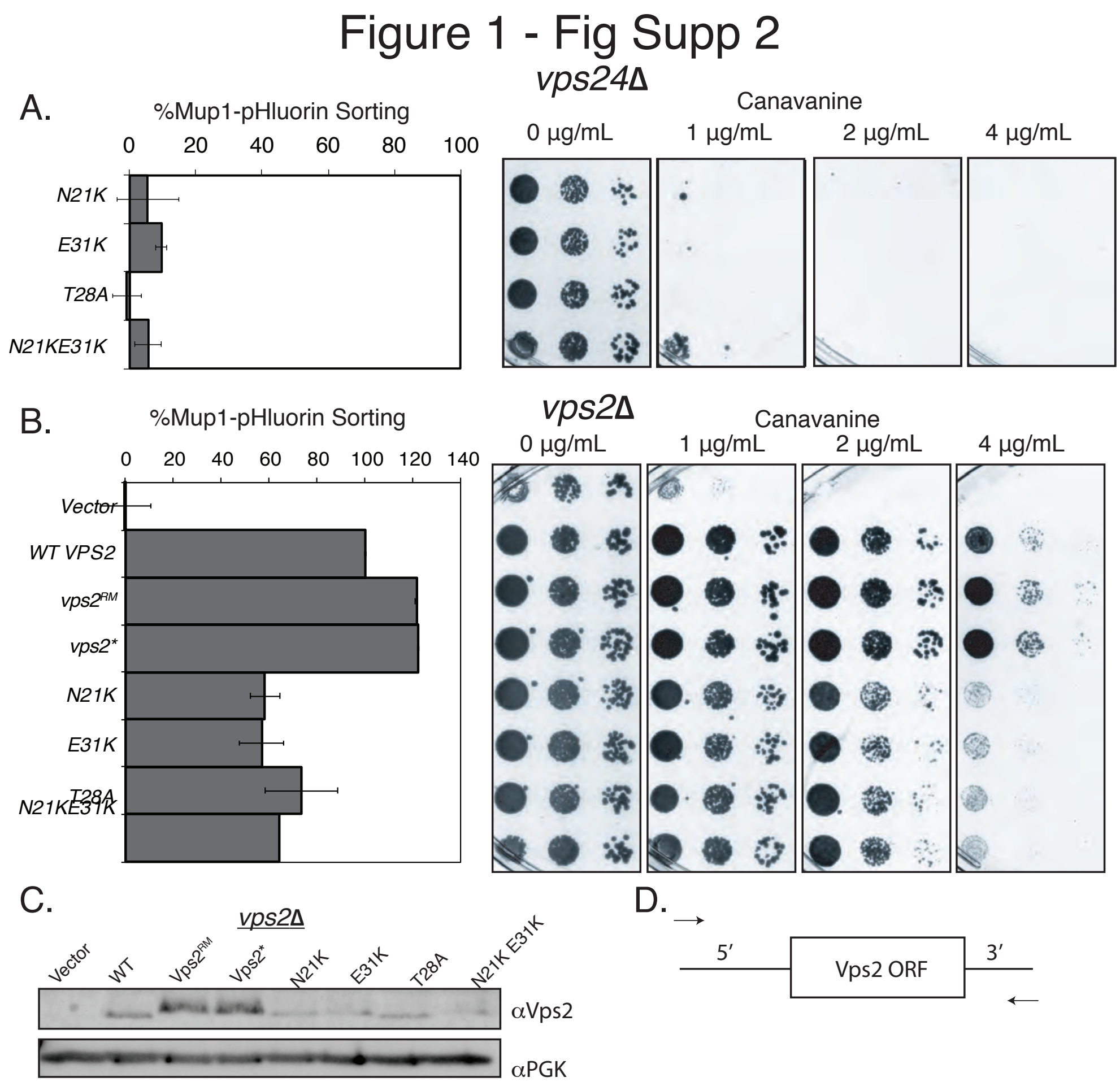

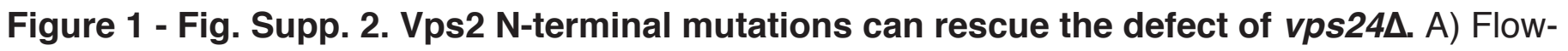
cytometry for Mup1-pHluorin sorting and canvanine sensitivity assay in vps24 $\Delta$ for the $\mathrm{N}$-terminal helix-1 mutations in Vps2 (compare with Fig. $1 \mathrm{C}$ ). In these constructs the promoters were endogenous, WT promoters. B) In a vps2 $\triangle$ background, the suppressors $v p s 2^{R M}$ and $v p s 2^{*}$ also have higher sorting capabilities in both Mup1-sorting assay and in canavanine sensitivity assay. The promoter regions of $v p s 2^{R M}$ and $v p s 2^{*}$ contains mutations, but other constructs are with WT promoters. C) Immunoblots of various Vps2 mutants, the same constructs as used in Fig. 1C, and Fig. 1 - Supp. 2 A and C. D) Design of the randomly mutagenized Vps2 plasmid - primers bind to the 5' and 3' UTR regions of Vps2. 


\section{Figure 1 - Fig Supp 3}

A.

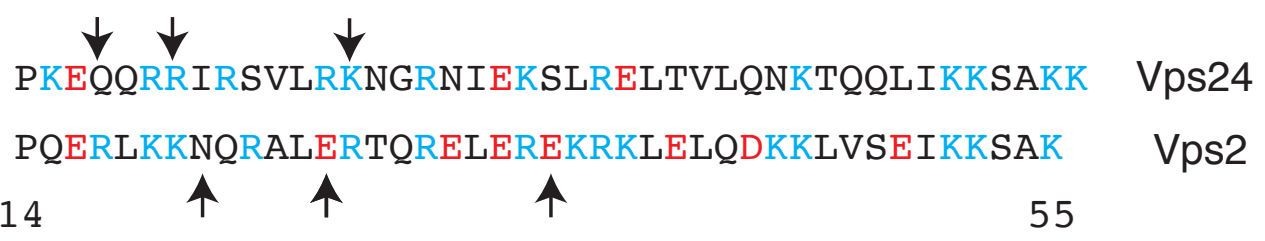

B.

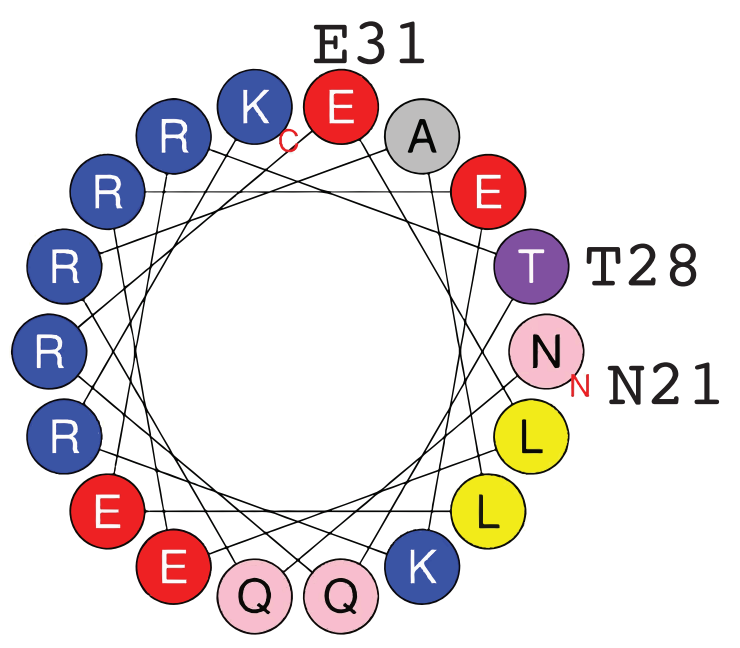

Figure 1 - Fig. Supp. 3. Helix-1 region of Vps2 is important for binding to Snf7. A) Sequence alignment of helices 1 of Vps24 and Vps2. Cyan-colored residues are basic amino acids, and red colors represent acidic amino acids. Arrows in Vps24 sequence point to mutations that rescue the defect of the snf $7^{1131 \mathrm{~K}}$ allele (Banjade et al., 2019). Arrows in Vps2 sequence represent the location of the mutations that rescue vps24 $\Delta$. B) Helical wheel representation of part of the helix-1 region of Vps2 (Heliquest). 
A.

A. $\quad$ vps24 2 vps2

\section{Figure 2}

\section{Canavanine}
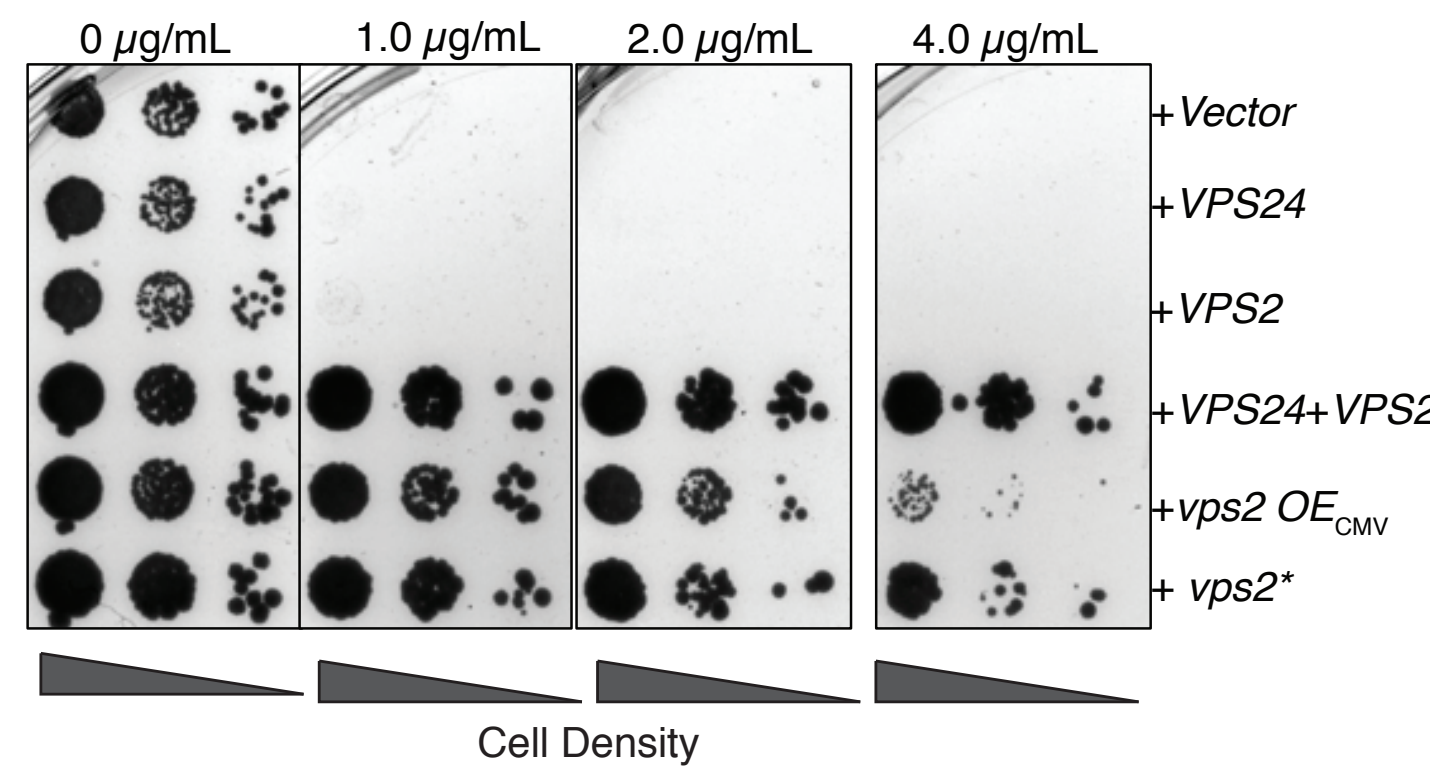

B.
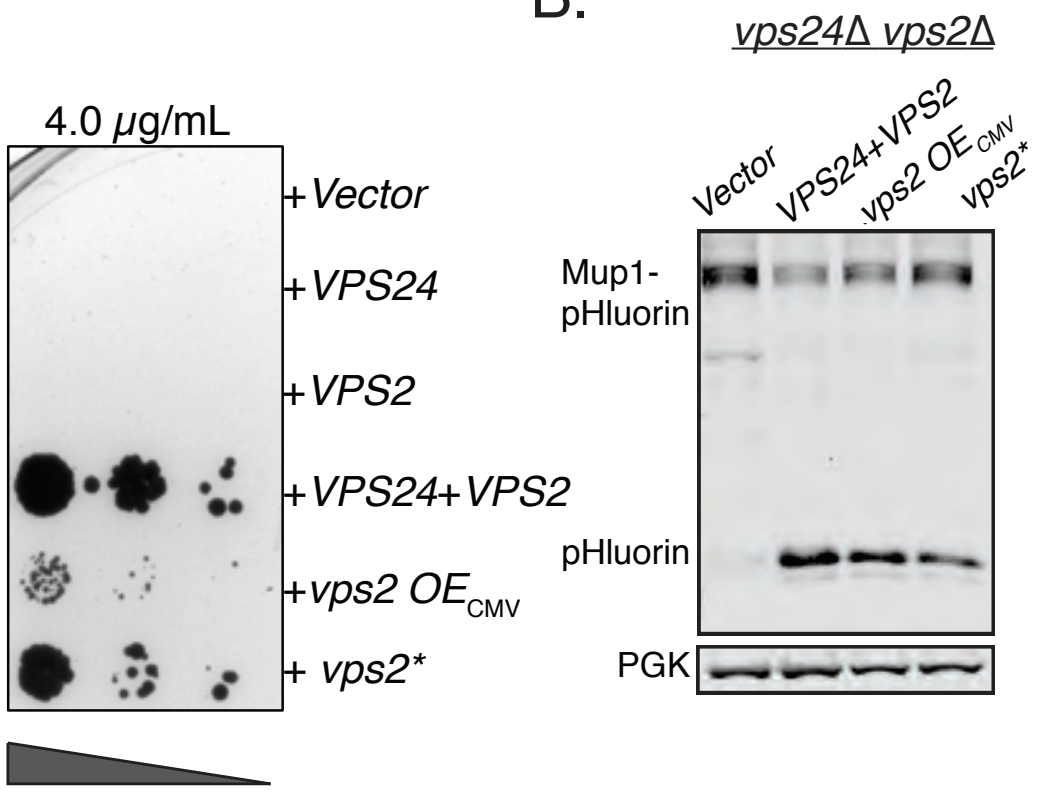

Cell Density

C.

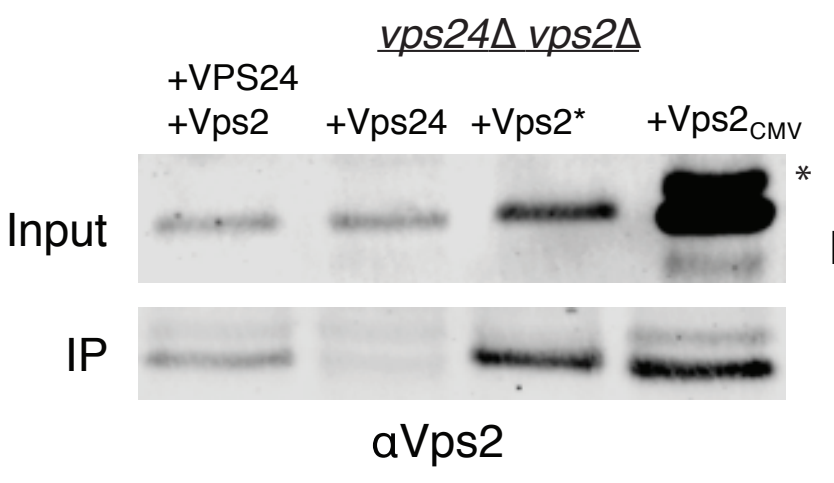

IP: Snf7, WB: Vps2

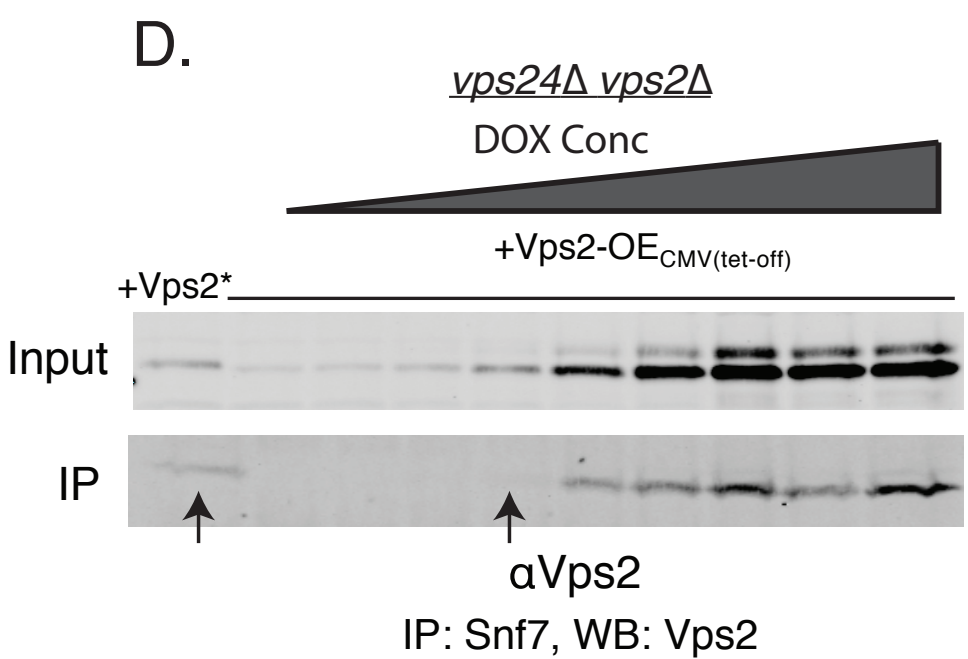

psestavs 24

P. Snf7, WB: Vps2

Figure 2. Properties of both Vps2 and Vps24 in a single Vps2 contruct. A) Canavanine sensitivity data in vps24 $v p s 2 \Delta$ with an overexpression of Vps2 (CMV-Tet system) or with Vps2*. B) Immunoblot for Mup1-pHluorin sorting upon overexpression of Vps2 (CMV) or with Vps2*. C) Co-immunoprecipitation of Snf7 with Vps2 (CMV) and Vps2*. D) Coimmunoprecipation experiments of Snf7 with Vps2 at various expression levels of Vps2 after titration of the tet-off operator with doxycycline. Arrows point to the relative binding to Snf at similar expression levels of Vps2 and Vps2*. In the gels, ${ }^{*}$ refers to an unknown modified form of Vps2. 


\section{Figure 3}

A.
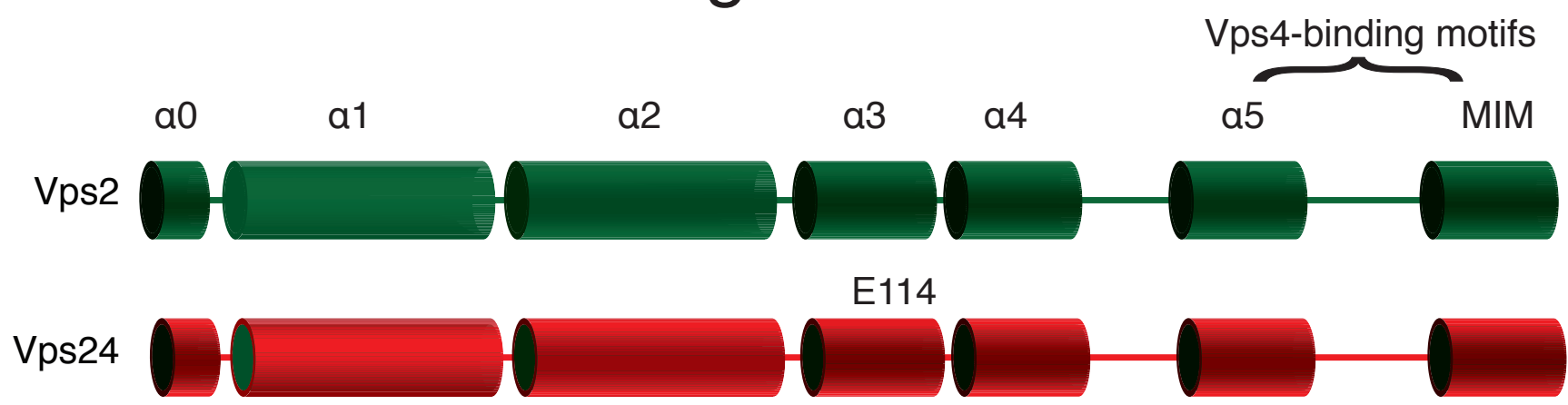

B.
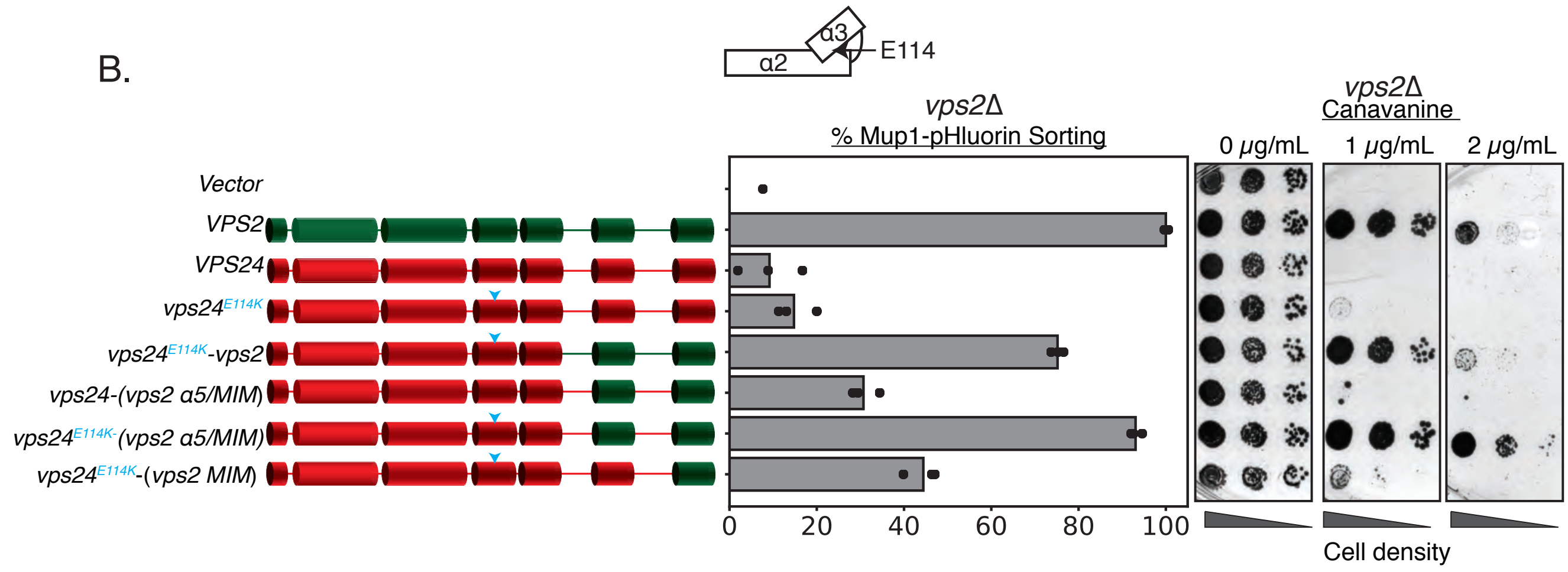

Figure 3. Simple modifications in Vps24 can be made to mimic Vps2. A) The domain organization of Vps2, highlighting the C-terminal region important for Vps4 binding. B) Left-panel denotes the chimeras made to replace regions of Vps2 onto Vps24. Cyan arrows in the helices are positions of the E114K mutation. Right panel represents Mup1-pHluorin sorting and canavanine-sensitivity assays. In this assay, the constructs were over-expressed under a CMV-promoter-Tetoff operator system, overexpressing Vps24 16 fold. 


\section{Figure 3 - Fig Supp 1}

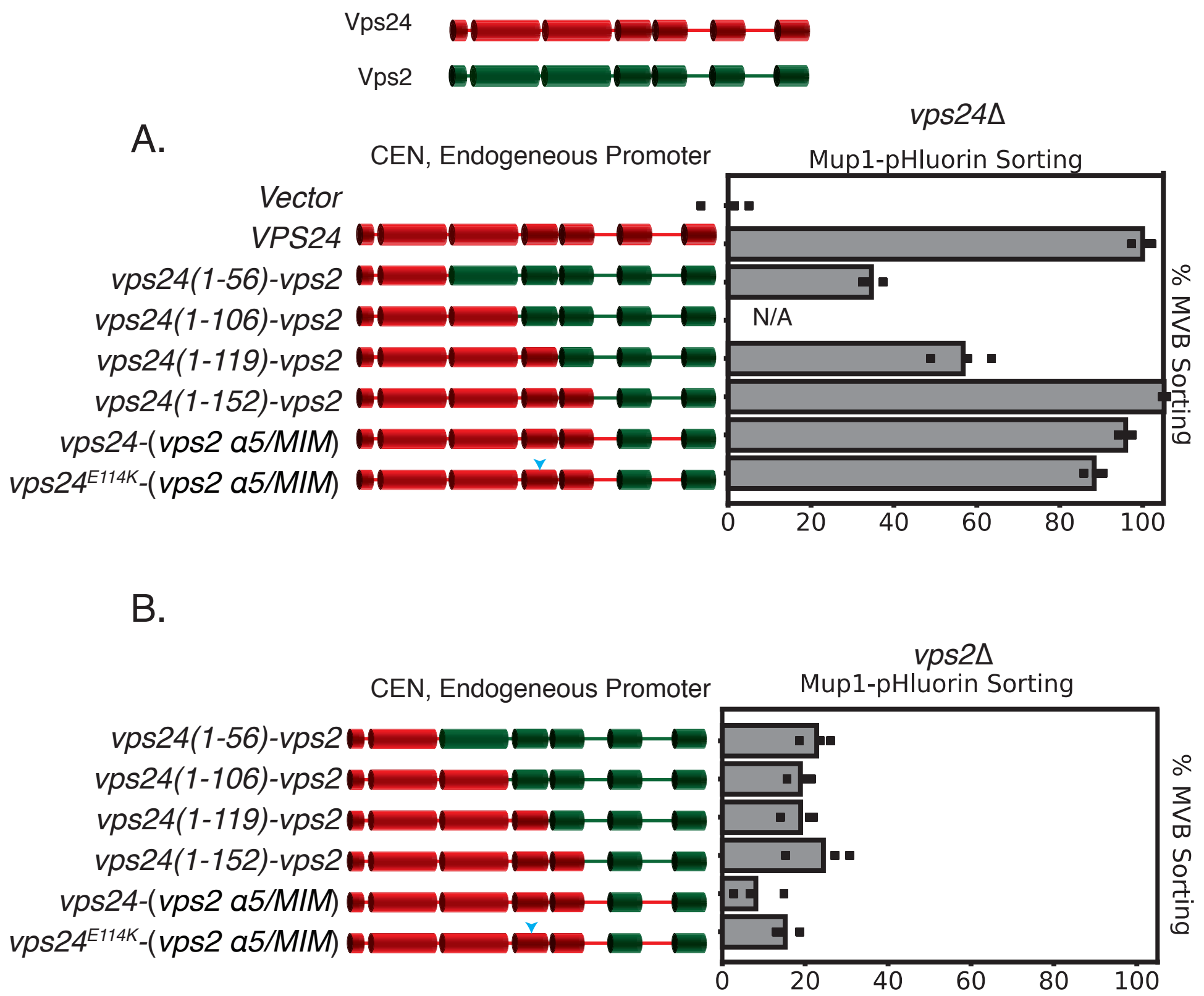

Figure 3 - Fig Supp. 1. Chimeras of Vps24-Vps2 are functional proteins. Top figure depicts the domain organization of Vps2 and Vps24. A) Mup-pHluoring sorting assay with several chimeras of Vps24-Vps2, showing that the replacement of the C-terminal regions of Vps2 onto Vps24 keeps the constructs functional. B) The same constructs as in (A) do not supress vps2 $\Delta$, as they are under endogeneous prometers. "CEN" represents denotation for centromeric plasmid. 


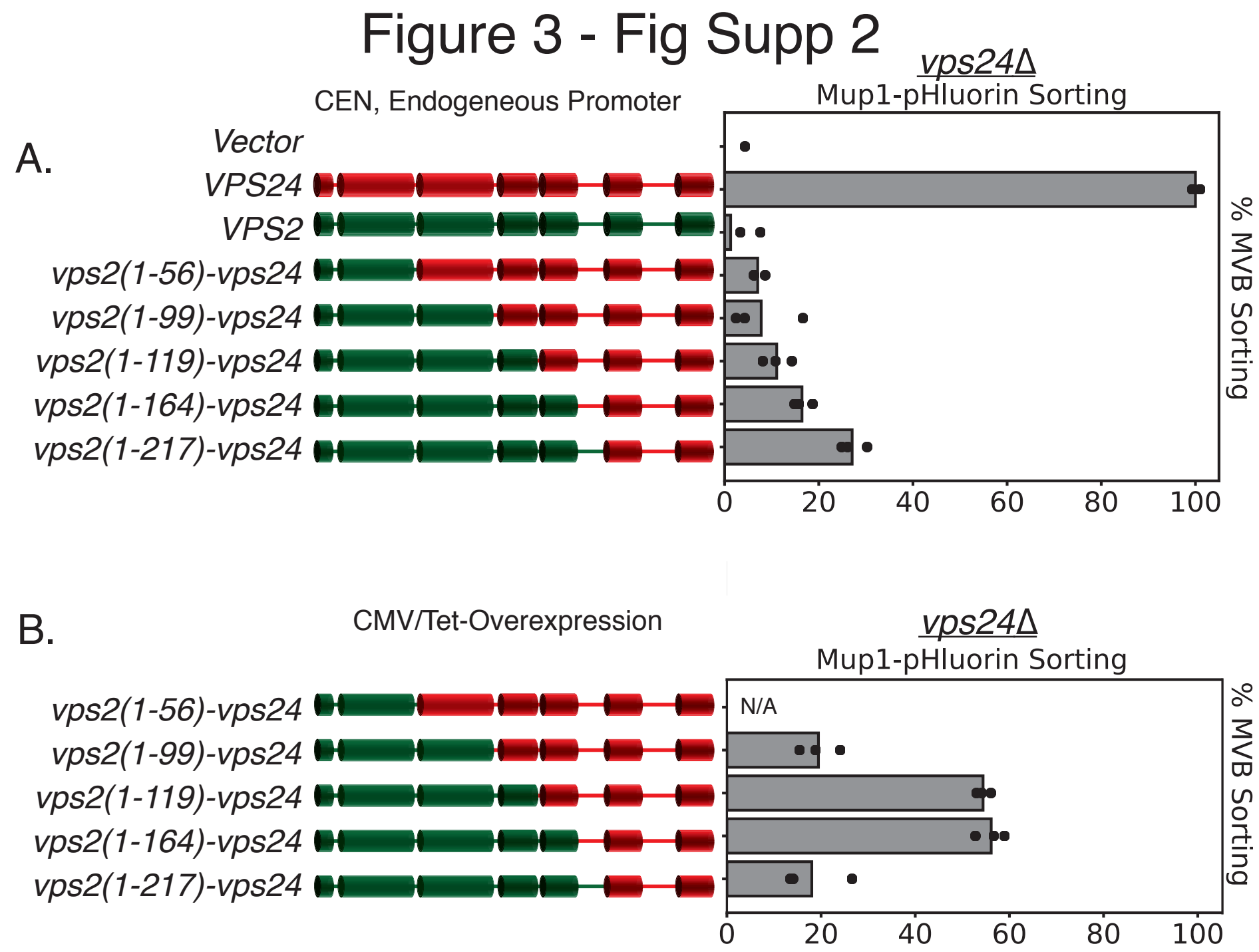

Figure 3- Fig. Supp. 2. Simple modifications in Vps24 can be made to mimic Vps2. A) Under the endogenous promoter and centromeric plasmid (CEN), various chimeras of Vps24-Vps2 do not support the sorting of Mup1-pHluorin, but some of the same constructs when overexpressed can rescue $v p s 24 \Delta(\mathrm{B})$. Note that the $\mathrm{N}$-terminus of Vps2 needs to be intact to mimic Vps24. Overexpression ( 16 fold) was achieved with a CMV-promoter, Tet-operator system. 

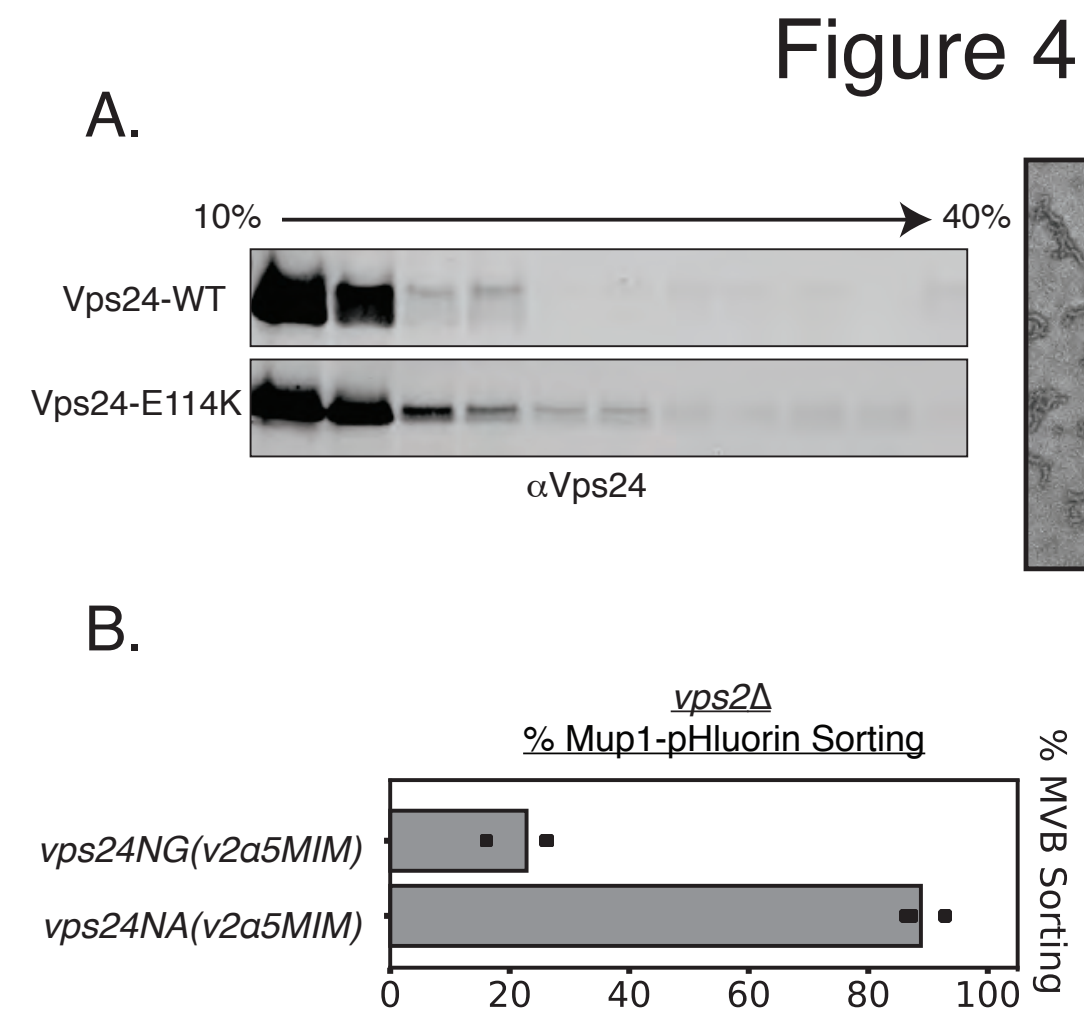

C.
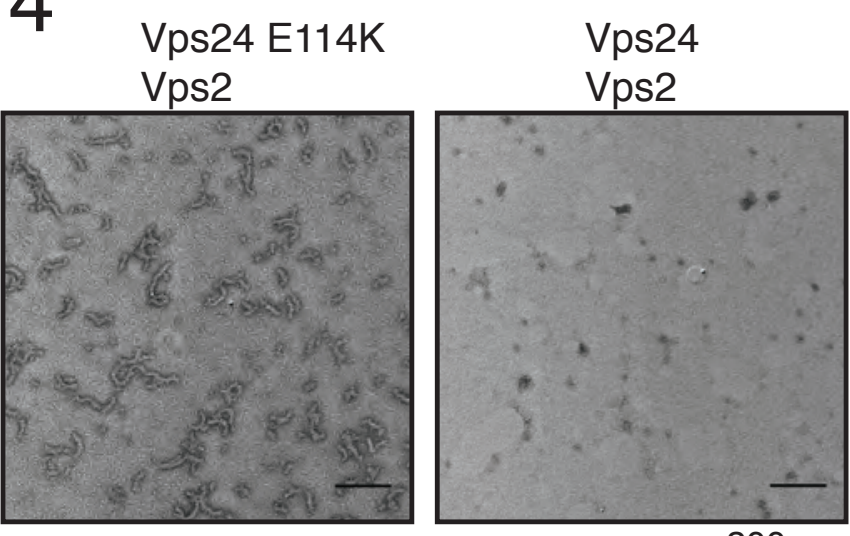

$200 \mathrm{~nm}$
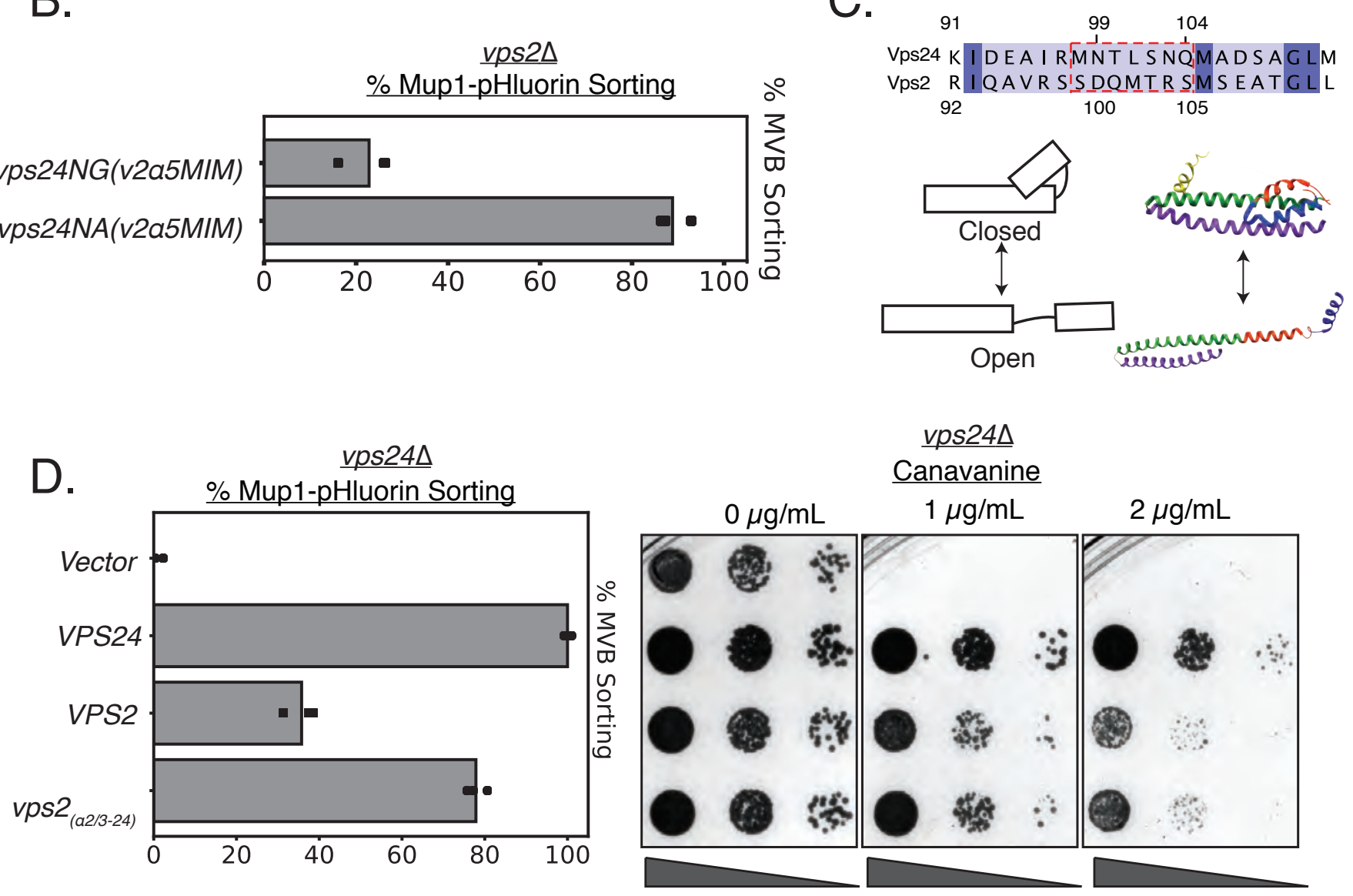

Figure 4. Vps24 and Vps2 may exhibit different conformations. A) Left: glycerol-gradient experiments with Vps24 and Vps24E114K suggests that the mutant can form higher molecular-weight species. Right: negative stain electron microscopy of Vps24 E114K or WT Vps24 at 1 $\mu \mathrm{M}$ each of the proteins in the presence of Vps2. B) Mup1-pHluorin assays with Vps24 mutations in the asparagines (N99 and N103) a2/a3 hinge region to Ala or Gly residues in constructs that have the Vps4-binding sites H5(helix-5) and MIM from Vps2 (V2). These constructs are expressed with the CMV-promoter, Tet-off system. See Fig. 3 for direct comparison with other Vps24 mutants and chimeras.C) Top: sequences of the a2/a3 hinge region of Vps24 and Vps2. Bottom left: Model showing the two conformations of ESCRT-III proteins. Structural model on the right is that of CHMP3(closed) (Bajorek, 2009) and of Snf7(open) (Tang, 2015). D) Mup1-pHluorin sorting and canavanine-sensitivity assays with overexpression of Vps2 (CMV-Tet) and with a mutant replacing the a2/a3 hinge region of Vps2 with that of Vps24 (also CMV-Tet system). 


\section{Figure 4 - Supp 1}
A.
Vps24 E114K Vps2

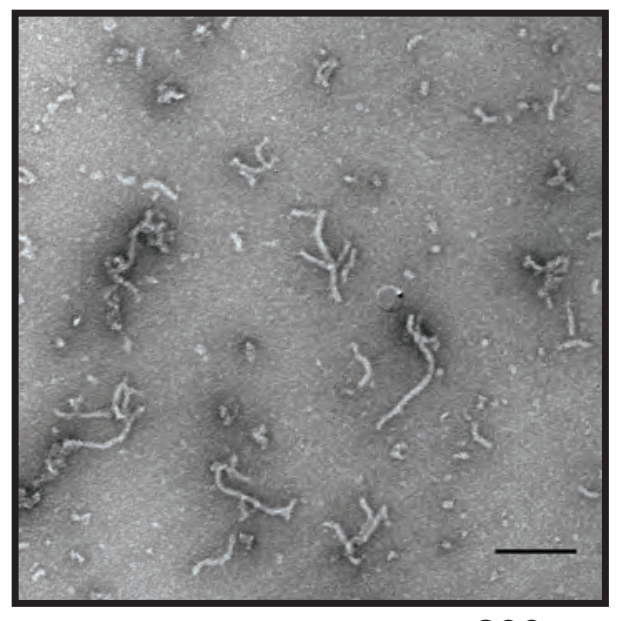

$200 \mathrm{~nm}$
Vps24 E114K Vps2

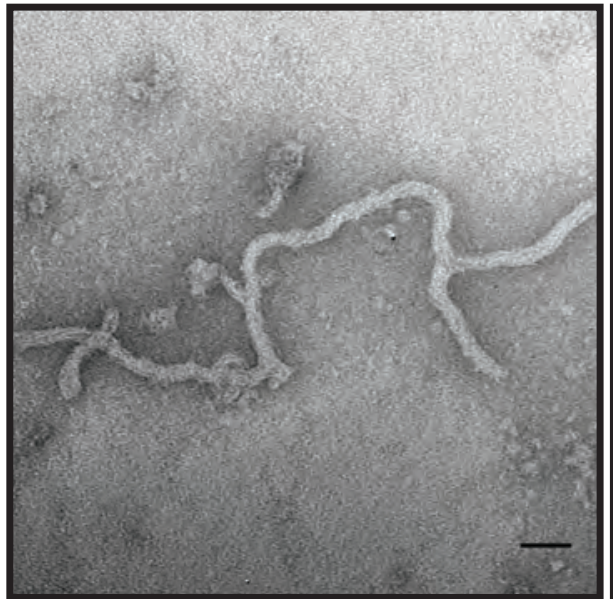

B.
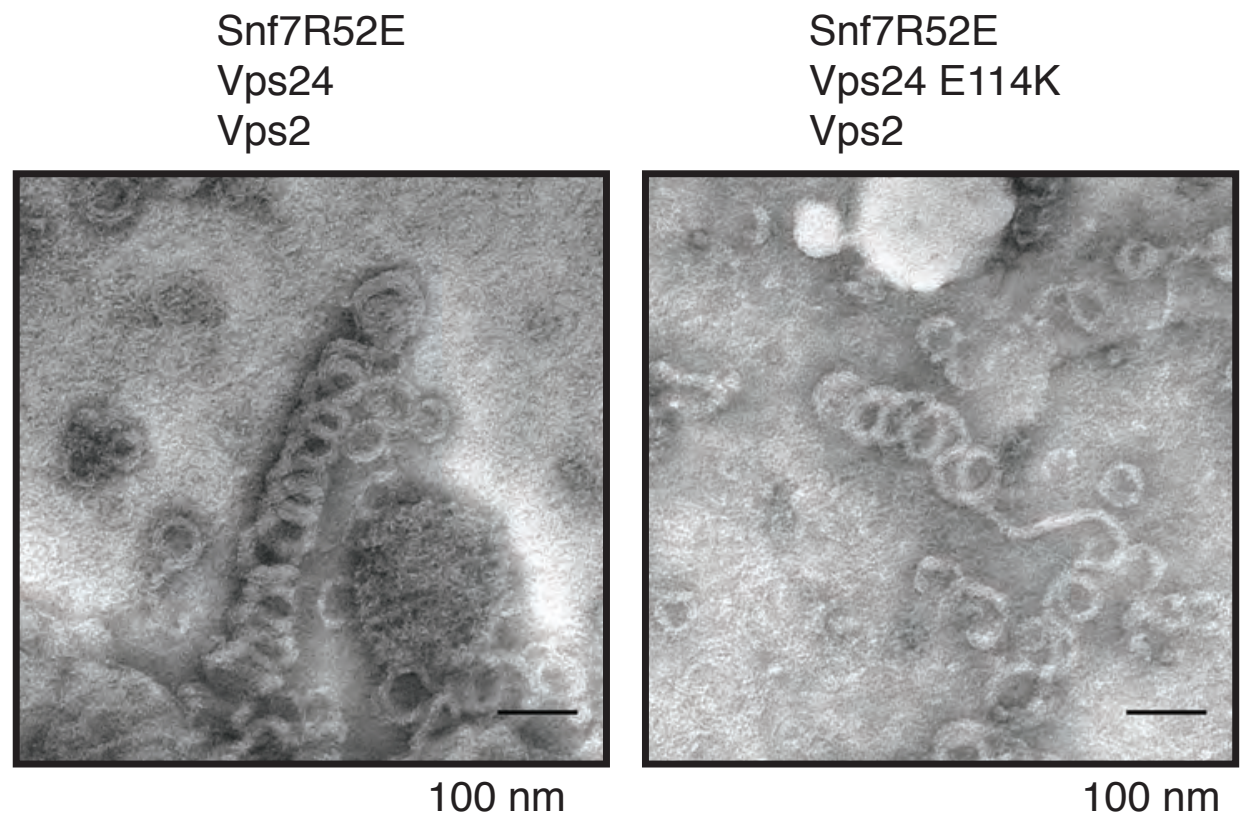

Figure 4- Fig. Supp 1. Vps24 (E114K) associates with Vps2. A) Electron microscopy images of Vps24E114K assembled with Vps2 at concentrations of $5 \mu \mathrm{M}$ each. Two images on the right are zoomed-in images of the same polymers. Vps24 E114K alone or Vps24 with Vps2 ) do not form such polymers. B) Vps24E114K mutant with Vps2 and Snf7-R52E still form 3D helices. Snf7-R52E is a mutant that has a lower critical concentraiton for polymerization as a higher fraction of this protein is in an open conformation (Henne et al., 2012). 


\section{Figure 4 - Fig Supp 2}

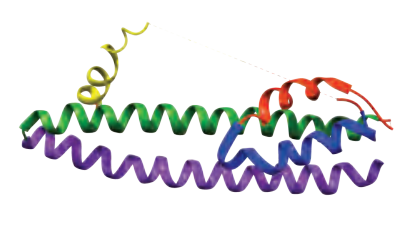

CHMP3 (PDB 3FRT)

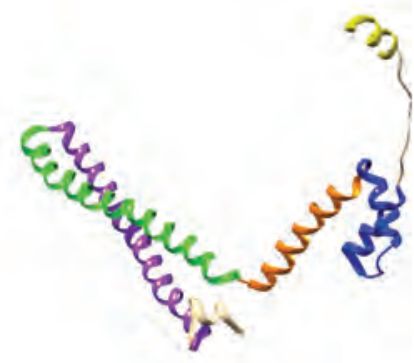

Vps24 (PDB 6ZH3)

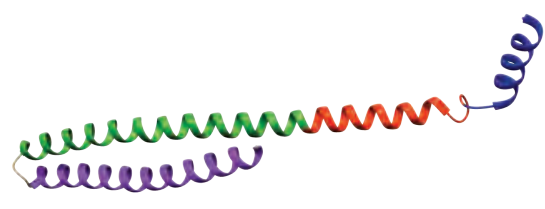

Snf7 (PDB 5FD7)

Figure 4- Fig. Supp 2. Structures of the autoinhibited CHMP3, and the filament forming conformations of Vps24 and Snf7. 


\section{Figure 5}

vps20

Can $0 \mu \mathrm{g} / \mathrm{mL}$

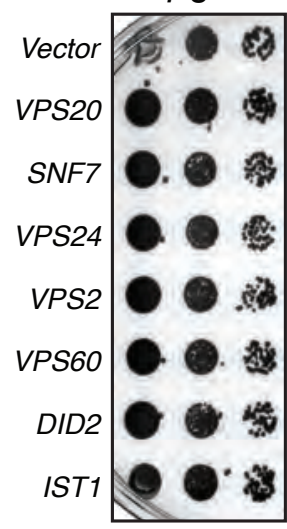

vps2 2

Can $\quad 0 \mu \mathrm{g} / \mathrm{mL} \quad 0.5 \mu \mathrm{g} / \mathrm{mL} \quad 1.0 \mu \mathrm{g} / \mathrm{mL}$

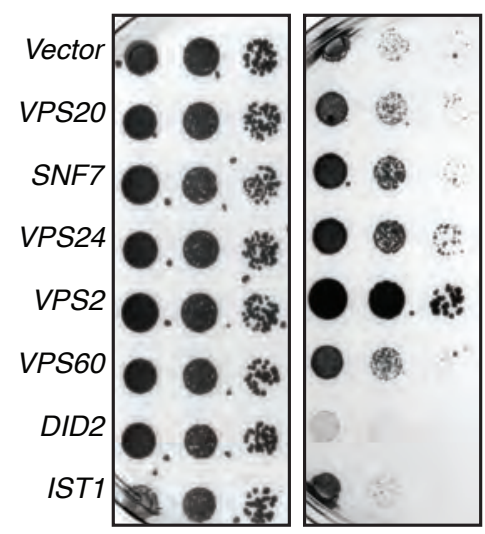

$\underline{s n f 7 \Delta}$

$0.5 \mu \mathrm{g} / \mathrm{mL} \quad 1.0 \mu \mathrm{g} / \mathrm{mL}$

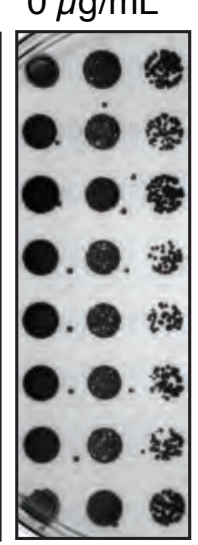

did $2 \Delta$

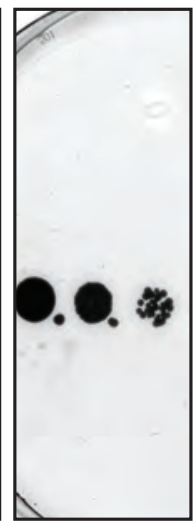

$0 \mu \mathrm{g} / \mathrm{mL}$

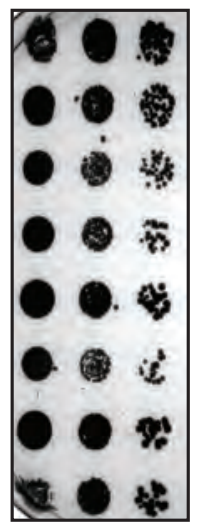

$0.5 \mu \mathrm{g} / \mathrm{mL} \quad 1.0 \mu \mathrm{g} / \mathrm{mL}$

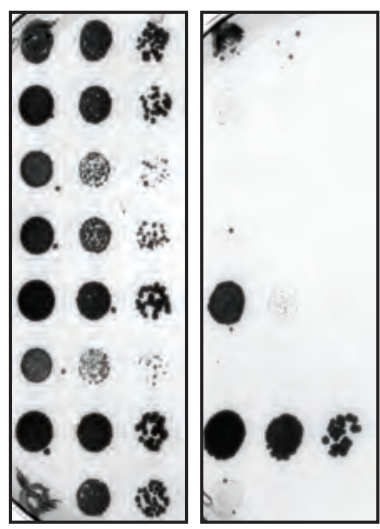

ist1 $\Delta$

Can $0 \mu \mathrm{g} / \mathrm{mL} \quad 0.5 \mu \mathrm{g} / \mathrm{mL} \quad 1.0 \mu \mathrm{g} / \mathrm{mL}$
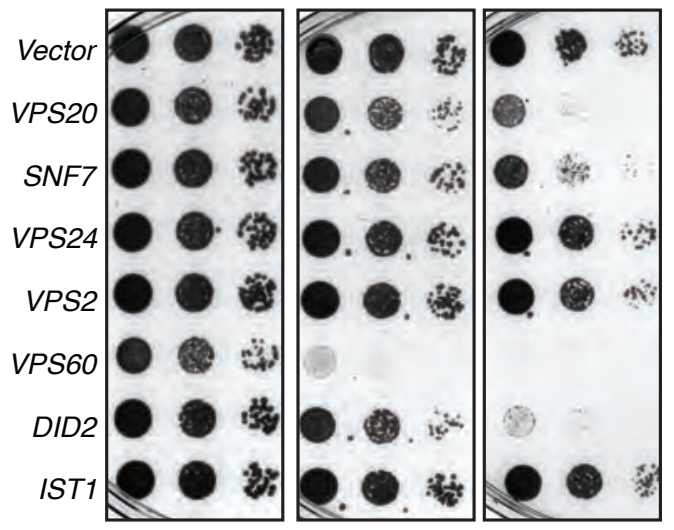

vps24ム

$0 \mu \mathrm{g} / \mathrm{mL} \quad 0.5 \mu \mathrm{g} / \mathrm{mL} \quad 1.0 \mu \mathrm{g} / \mathrm{mL}$

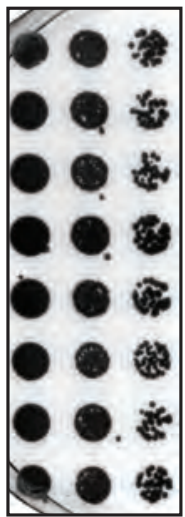

vps604

$0 \mu \mathrm{g} / \mathrm{mL} \quad 0.5 \mu \mathrm{g} / \mathrm{mL} \quad 1.0 \mu \mathrm{g} / \mathrm{mL}$

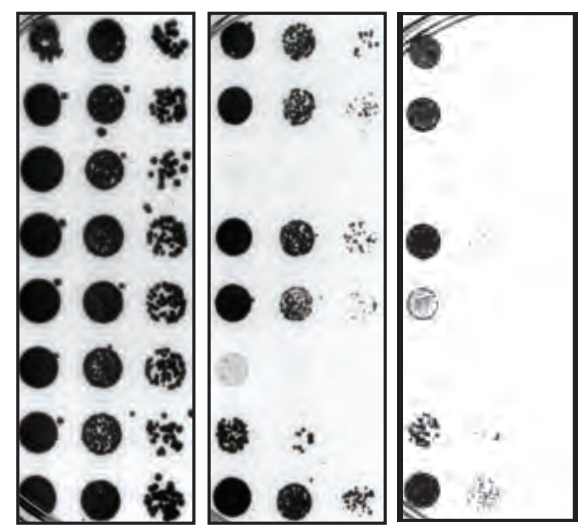

Cell Density

Figure 5. Overexpressing ESCRT-III proteins in the background of other ESCRT-III mutants show selective rescue phenotypes. In the annotated mutants, ESCRT-III proteins were expressed with a CMV-promoter/Tet-operator system, and plated in canavanine-containing plates. Vps2 overexpression can rescue the defect of vps24 $\Delta$. Vps2 overexpression in a did2 $\Delta$ partially rescues canavanine sensitivity. Vps60 overexpression appears to be dominant negative. 


\section{Figure 5 - Figure Supplement 1}
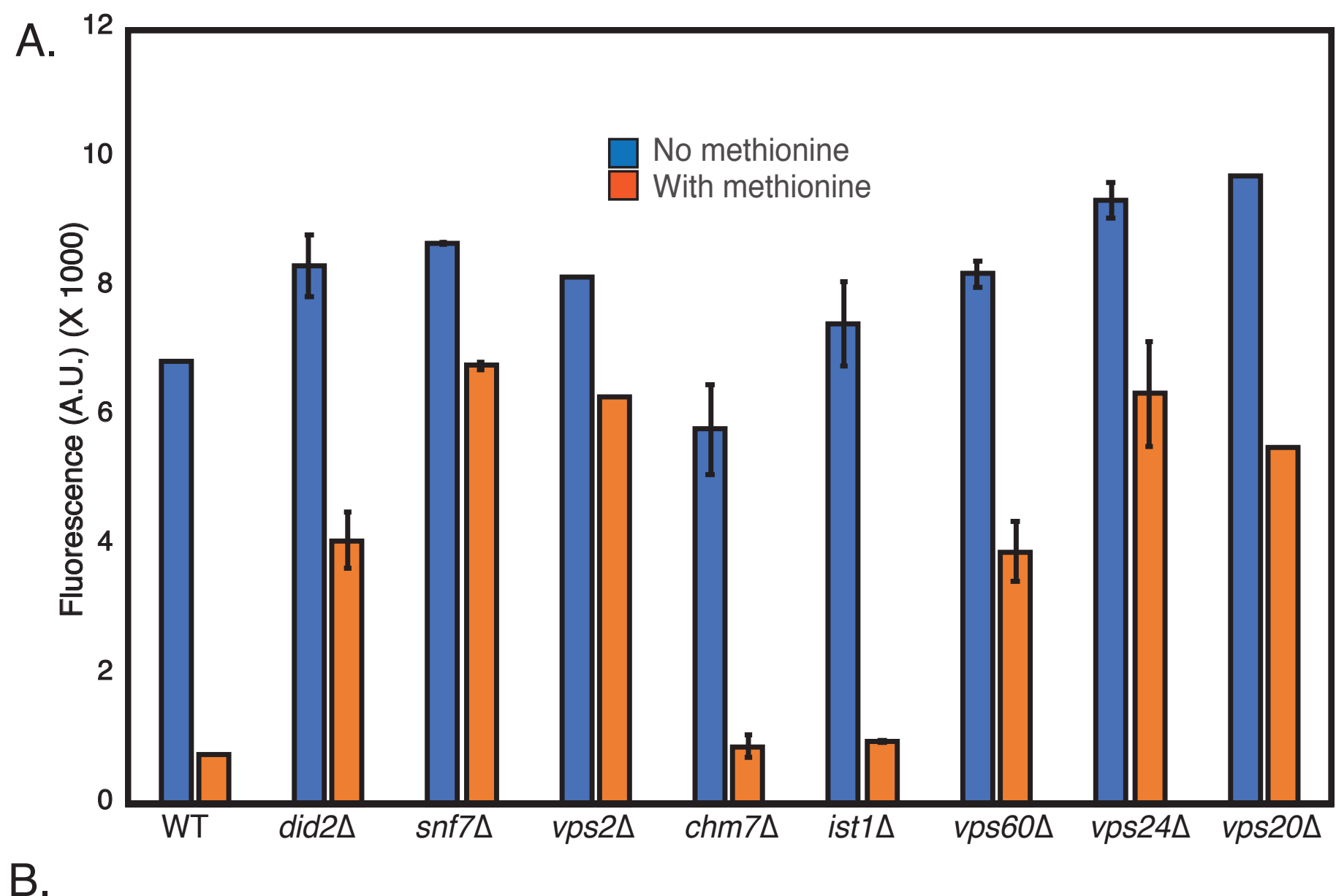

B.

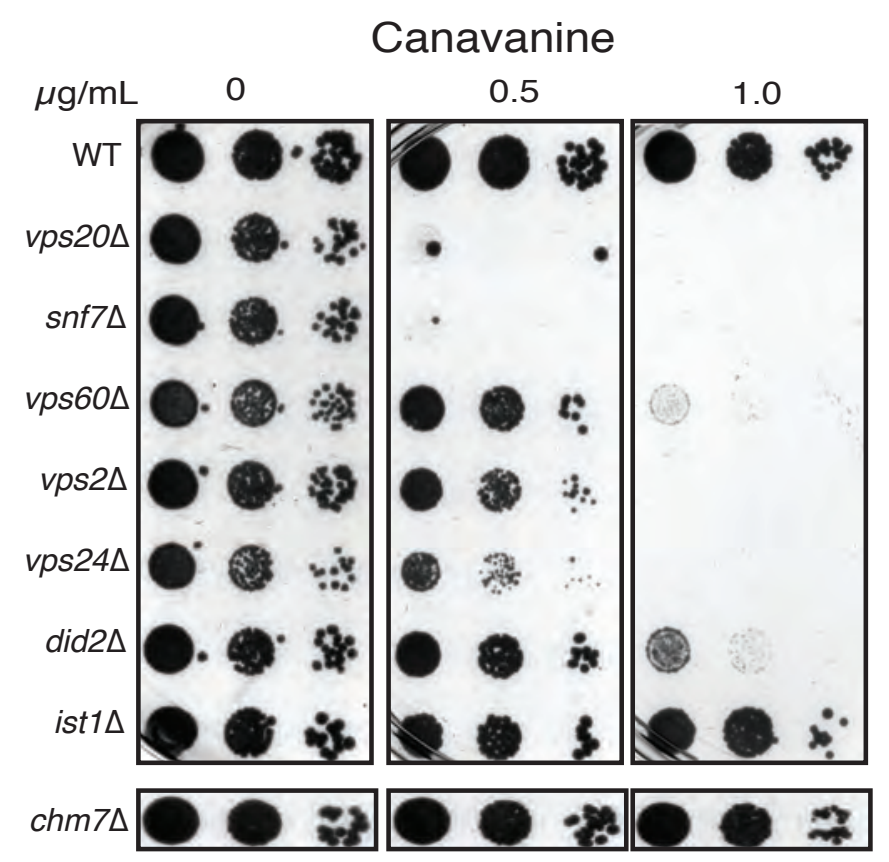

Figure 5 - Figure Supplement 1. A) Relative effects of all ESCRT-III mutants for defects in cargo sorting, using Mup1-pHluorin assay. Fluorescence of 100,000 cells were measured after 90 minutes of adding of $20 \mu \mathrm{g}$ methionine. B) Canavanine sensitivity assays of the ESCRT-III mutants. 


\section{Figure 6}

A.

$\mid \begin{aligned} & \text { Vps20 (CHMP6) } \\ & \text { Snf7 (CHMP4) } \\ & \text { Vps60 (CHMP5) }\end{aligned}$

$\mid \begin{gathered}\text { Vps24 (CHMP3) } \\ \text { Vps2 (CHMP2) } \\ \text { Did2 (CHMP1) }\end{gathered}$

Ist1 (IST1)

Chm7 (CHMP7)

(C-terminus)
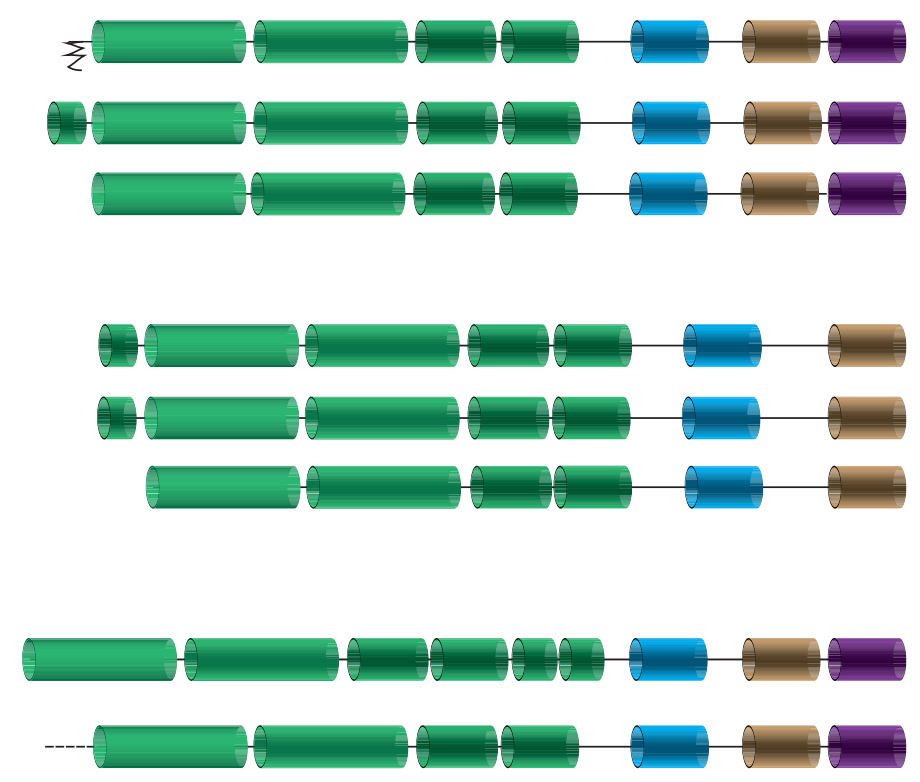
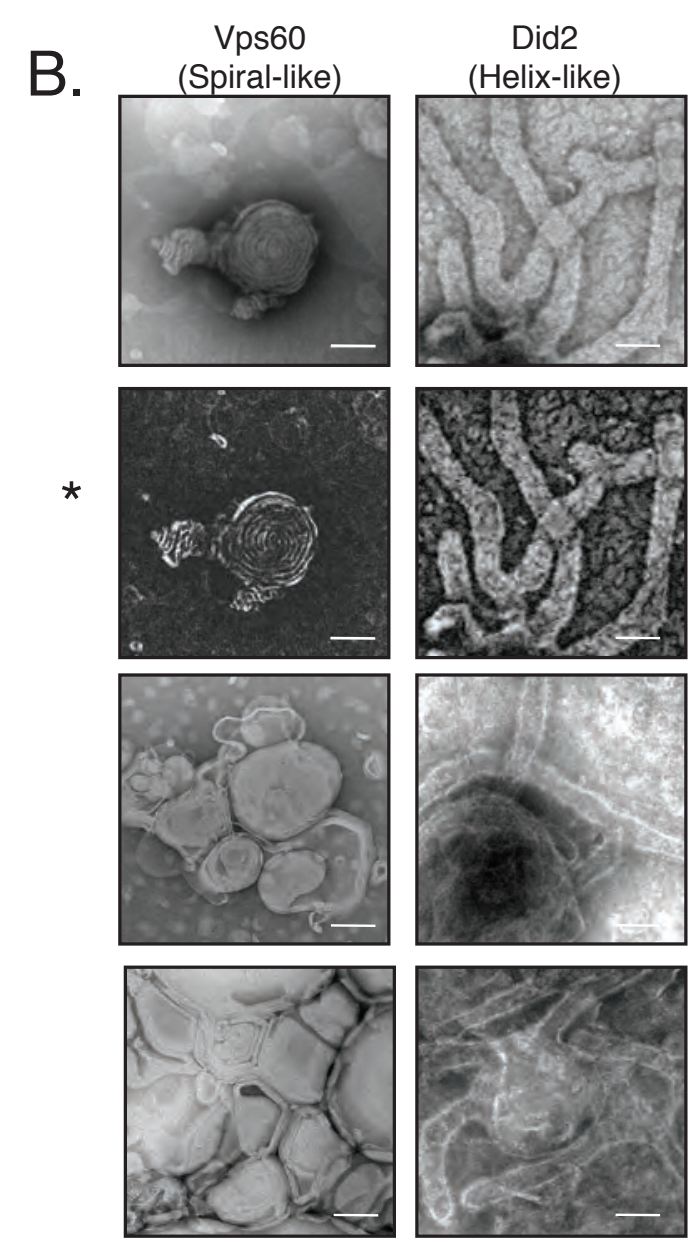

Bar $200 \mathrm{~nm}$ vps60\$

a0 a1 a2 a3 a4 a5 MIM a6

Canavanine $(\mu \mathrm{g} / \mathrm{mL})$

12

vps60
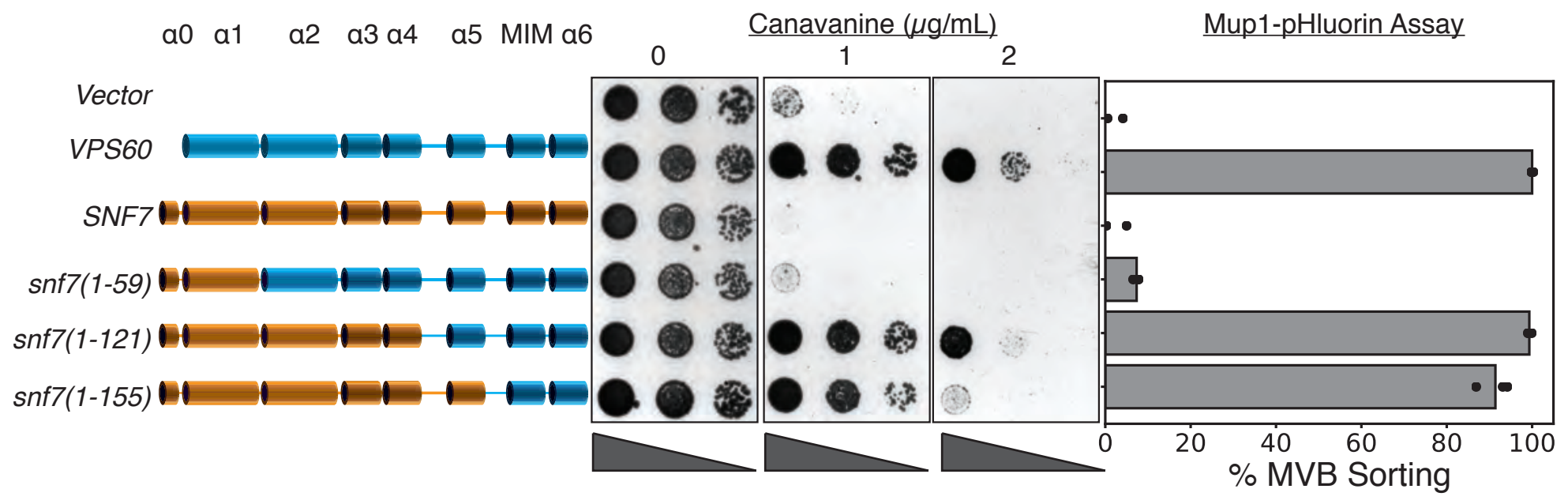

Figure 6 - Vps60 possesses features of Snf7. A) Domain subunits of the eight ESCRT-III proteins in yeast. The mammalian names are in parentheses. B) Electron microscopy images of $1 \mu \mathrm{M} \mathrm{Vps60}$ or $1 \mu \mathrm{M}$ Did2 on lipid monolayers, incubated for 1 hour. Bar is $200 \mathrm{~nm}$ each. Top two images are different constrast-adjusted depictions of the same image. C) Domain swaps from Snf7 onto Vps60 can rescue the defects of canavanine senstivity and Mup1-pHluorin sorting in a vps60 strain. 


\section{Figure 6 - Fig Supp 1}
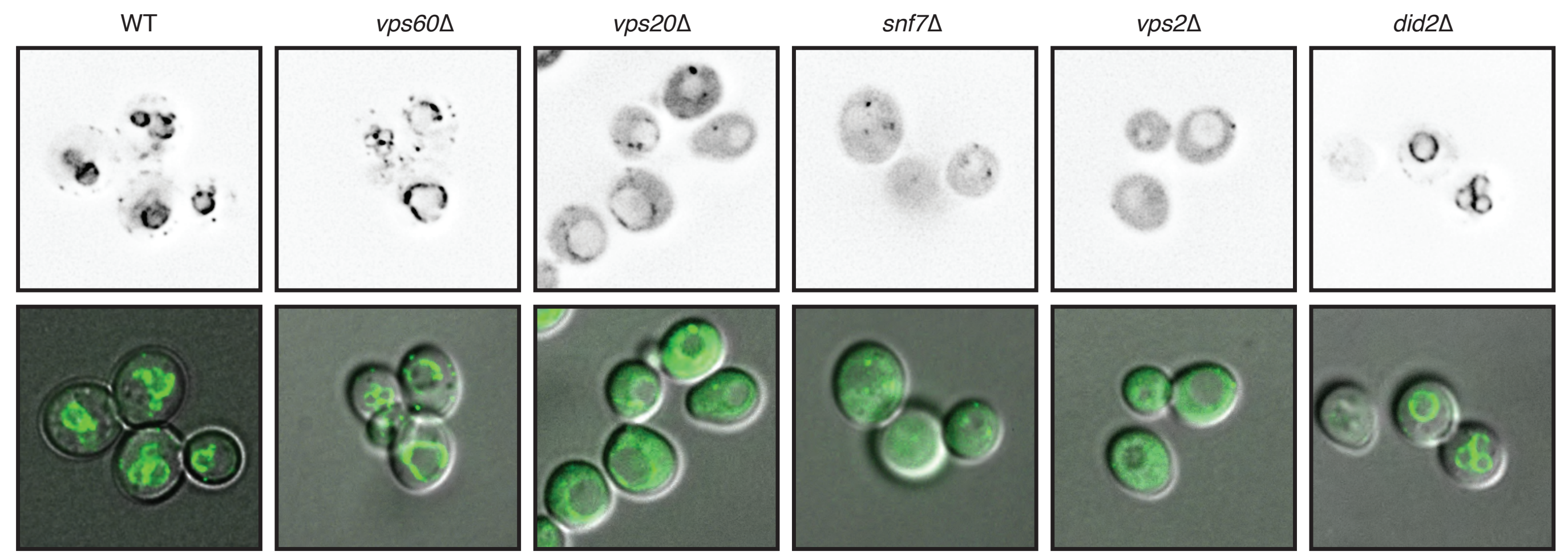

\section{VPS60-GFP}

Figure 6 - Fig. Supp. 1. Localization of Vps60-GFP in different ESCRT-III mutants. Top panel represents grayscaled GFP fluorescence and bottom panel represents a merge of GFP and DIC channels. While in WT strains VPS60-GFP primarily localizes to punctae (membranes), in various mutants the cytoplasmic signal is increased. 


\section{Figure 7}

A.

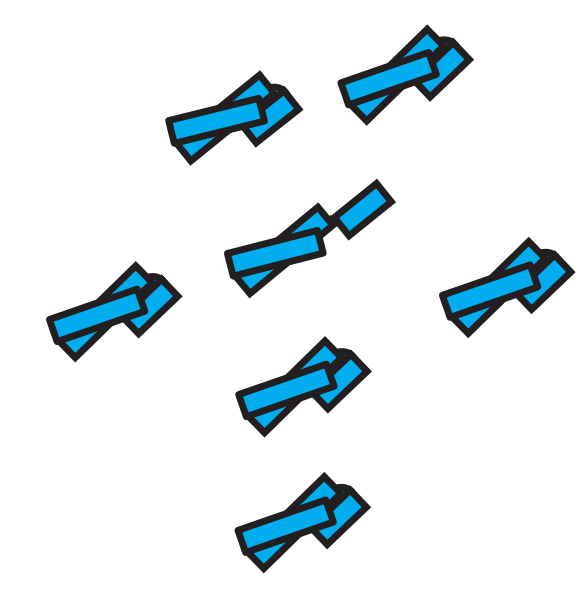

Vps24 (Higher closed population)

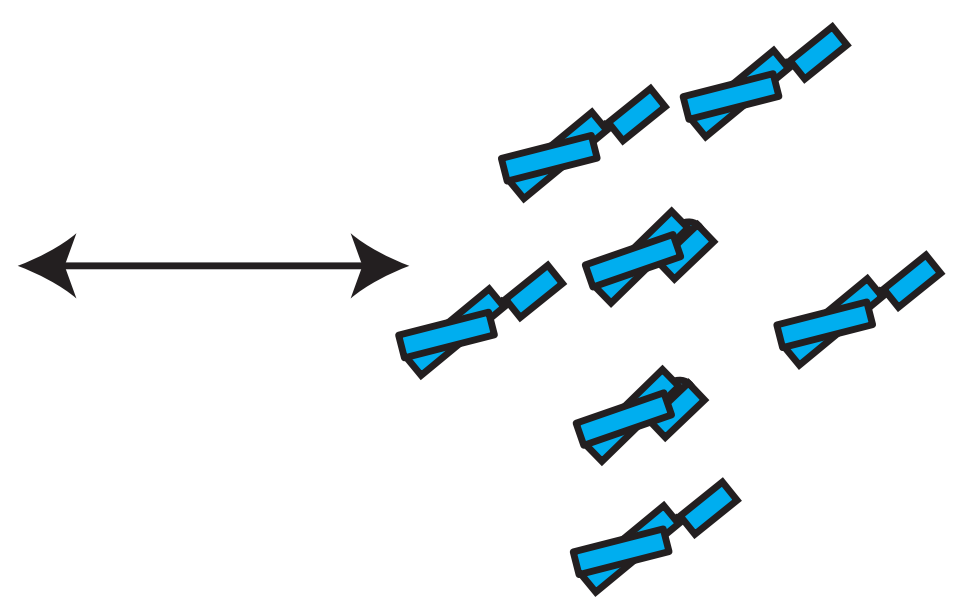

Vps2 (Higher open population)

B. ESCRT-III Subunits Structural Organization N-terminal Helical Bundle (Core Polymeric Unit)

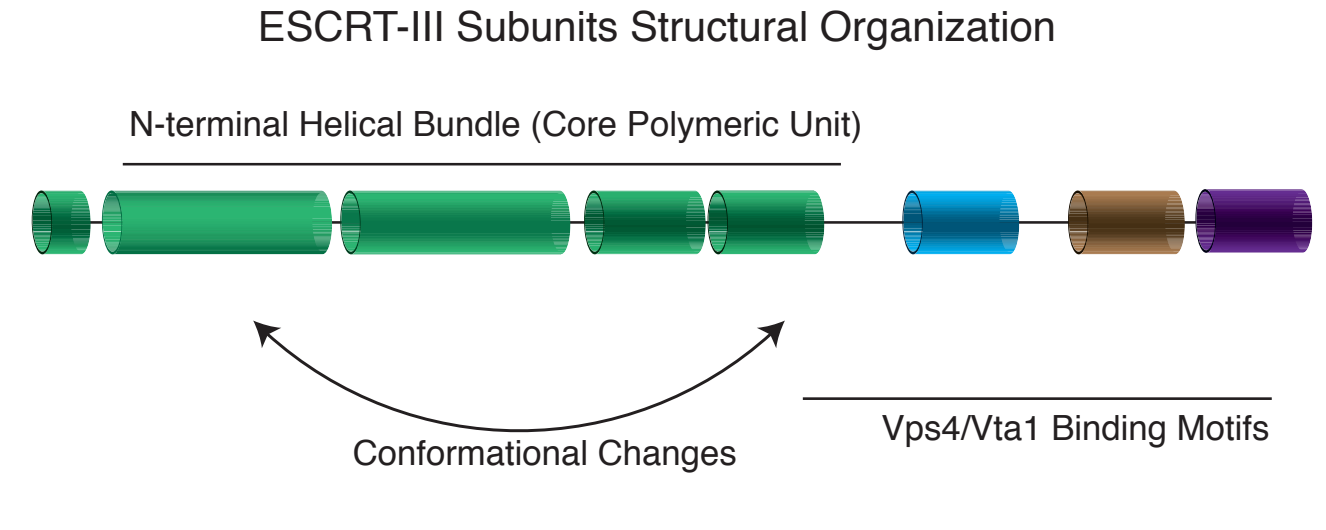

C.

i. Spiral formation

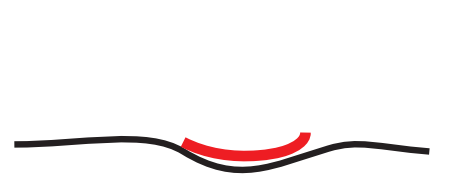

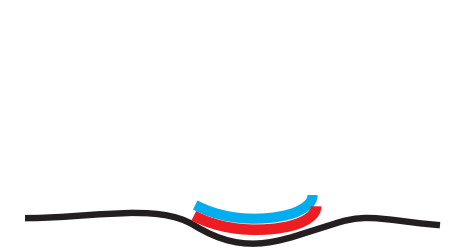

ii. Lateral association and helix formation through sequential polymerization of associated ESCRT-III poroteins
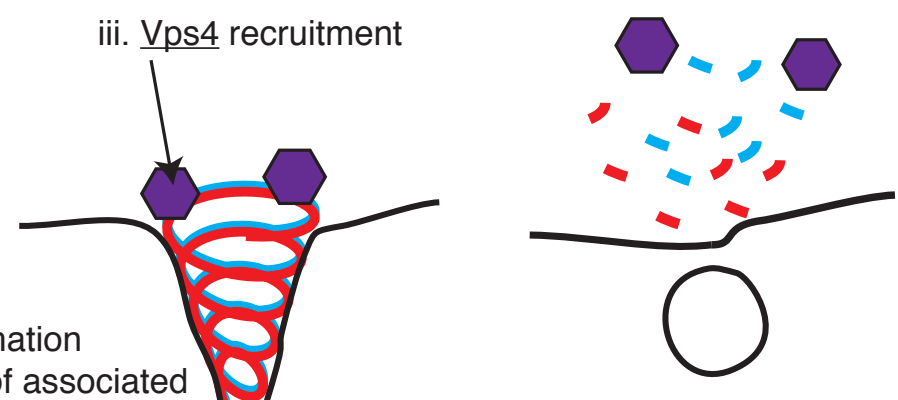

Vps4 recruitment leads to polymer remodeling and vesicle scission

Figure 7. ESCRT-III assembly principles. A) Vps24 and Vps2 are structurally similar proteins that may consist of two different populations of open and closed conformations. Switching between the two conformations mimic each other. B) The domain organization of ESCRT-III subunits and the variousfunctional parts of the structures/sequence. C) The minimal features of ESCRT-III assembly may involve spiral formation, lateral association between copolymers that induce helicity, and recruitment of a disassembly factor such as Vps4. 